\title{
GLOBAL GENERATION OF PLURICANONICAL AND ADJOINT LINEAR SERIES ON SMOOTH PROJECTIVE THREEFOLDS
}

\author{
LAWRENCE EIN AND ROBERT LAZARSFELD
}

\section{CONTENTS}

Introduction

0 . Notation and conventions

1. Kawamata-Viehweg vanishing and Reider's theorem

2. A theorem of Reider type for $\mathbb{Q}$-divisors on a surface

3. Main theorem: statement and set-up of proof

4. The case of an exceptional minimizing component

5. Nonexceptional minimizing components

6. Fujita's conjecture on global generation References

\section{INTRODUCTION}

The purpose of this paper is to show how the cohomological techniques developed by Kawamata, Reid, Shokurov, and others lead to some effective and practical results of Reider-type on freeness of linear series on smooth complex projective threefolds.

In recent years, there has been a great deal of interest in the geometric properties of pluricanonical and adjoint linear systems on surfaces and higherdimensional varieties. Among other things, one wants to understand as explicitly as possible when the systems in question are base-point free or very ample. Modern work in this area goes back to Kodaira [Kod] and Bombieri [Bmb], who studied pluricanonical maps of surfaces of general type. More recently, many of their results have emerged as special cases of a celebrated theorem of Reider [Rdr]. Reider uses vector bundle techniques to show that, if $B$ is a nef line bundle on a surface $X$ such that $B^{2} \geq 5$, then $K_{X}+B$ is globally generated unless there exist certain special curves $C \subset X$ such that $B \cdot C \leq 1$; he also obtains analogous criteria for $K_{X}+B$ to be very ample. A cohomological approach to these theorems, based on Miyaoka's vanishing theorem for Zariski decompositions, was given by Sakai [Sak2].

Received by the editors September 20, 1992.

1991 Mathematics Subject Classification. Primary 14F; Secondary 32F.

The first author was partially supported by NSF Grant DMS 91-05183.

The second author was partially supported by NSF Grant DMS 89-02551. 
In higher dimensions, naturally enough, much less is known. For adjoint linear series, a famous conjecture of Fujita [Fuj1] asserts that if $L$ is an ample bundle on a smooth projective variety $X$ of dimension $n$, then $K_{X}+(n+1) L$ is base-point free and $K_{X}+(n+2) L$ is very ample. For surfaces this follows from Reider's theorem, but in general it still seems out of reach. However in a real breakthrough Demailly [Dem] introduced some beautiful new analytic ideas leading to the first explicit conditions to guarantee that $K_{X}+L$ is globally generated or very ample. His statement involves the intersection numbers of $L$ with subvarieties of $X$ and a constant in effect measuring (with respect to $L$ ) the curvature of $X$. He deduces for instance that $2 K_{X}+12 n^{n} L$ is very ample as soon as $L$ is ample. In a somewhat different direction, Oguiso $[\mathrm{Og}]$ recently proved that if $L$ is an ample line bundle on a Calabi-Yau threefold $X$ (so that $K_{X}=\mathscr{O}_{X}$ ), then $20 L$ is globally generated and $60 L$ is very ample.

Concerning pluricanonical series, Kawamata $[\mathrm{K}]$ and Shokurov $[\mathrm{S}]$ proved that if $X$ is a smooth minimal projective variety of general type (so $K_{X}$ is numerically effective), then $\mathscr{O}_{X}\left(m K_{X}\right)$ is globally generated for all $m \gg 0$. For threefolds, Benveniste [Ben] showed that one can take $m \geq 34$, and Reid [Rd] also obtained some explicit statements (see also [Mat]). In the situation of the theorem of Kawamata-Shokurov, another conjecture of Fujita [Fuj2] predicts that $\left|m K_{X}\right|$ is free as soon as $m \geq n+2$. The proofs of the known results revolve around the Kawamata-Viehweg vanishing theorem for $\mathbb{Q}$-divisors. These cohomological techniques were developed by Kawamata, Reid, Shokurov, and others in connection with the minimal model program initiated by Mori. We refer the reader to [CKM] or [KMM] for an overview and further references. Our own interest in these questions grew out of the observation that one could use the approach of Kawamata-Reid-Shokurov to prove special cases of Reider's theorem. We will give the argument in $\S 1$. Kollár [Kol2] then showed that one could use these methods to give explicit estimates, in the spirit of Demailly, in all dimensions. His results imply, for instance, that on a smooth minimal $n$-fold of general type, $\left|2(n+2)(n+2) ! K_{X}\right|$ is base-point free and that $H^{0}\left(X, K_{X}+m B\right) \neq 0$ when $m>\left(\begin{array}{c}n+1 \\ 2\end{array}\right)$ for a big and nef line bundle $B$ on any smooth $n$-fold $X$.

While the existing effective statements are very interesting from a theoretical point of view, most of the constants that appear are very large. So it is natural to ask whether one can obtain results of a more "practical" nature, in the spirit of Reider's work or Fujita's conjectures. In the present paper we deal only with global generation on threefolds, but we aim for statements which, while generally not optimal, are at least in the right ballpark.

We start with an analogue of Reider's Theorem.

Theorem 1. Let $B$ be $a$ big and nef divisor on a smooth complex projective threefold $X$, and let $x \in X$ be a fixed point. Assume that $B^{3} \geq 92$ and

$$
\begin{array}{ll}
B \cdot C \geq 3 & \forall \text { curves } C \subset X \text { with } C \ni x, \\
B^{2} \cdot S \geq 7 & \forall \text { surfaces } S \subset X \text { with } S \ni x .
\end{array}
$$

Then $K_{X}+B$ is free at $x$, i.e., $\mathscr{O}_{X}\left(K_{X}+B\right)$ has a section which is nonvanishing at $x$. Moreover, if $B^{3} \gg 0\left(\right.$ e.g., $\left.B^{3} \geq 1000\right)$, then the same conclusion holds 
with the second inequality replaced by $B^{2} \cdot S \geq 5$.

One can use $\mathbb{P}^{2}$ bundles over a curve to see that, if there are curves $C$ with $B \cdot C \leq 2$ and surfaces $S$ with $B^{2} \cdot S \leq 4$, then it can happen that $B^{3}$ is arbitrarily large but $H^{0}\left(X, \mathscr{O}_{X}\left(K_{X}+B\right)\right)=0$. Theorem 1 is deduced from the following more general numerical criterion, in terms of the intersection numbers $B^{3}, B^{2} \cdot S$, and $B \cdot C$, for $\mathscr{O}_{X}\left(K_{X}+B\right)$ to be free at a point $x$ :

Theorem 1*. In the situation of Theorem 1, suppose that $\sigma_{3}, \sigma_{2}, \sigma_{1}>0$ are rational numbers such that $B^{3}>\sigma_{3}^{3}>3^{3}, B^{2} \cdot S>\sigma_{2}^{2}$, and $B \cdot C>\sigma_{1}$ for all surfaces $S \ni x$ and curves $C \ni x$. If

$$
\sigma_{2} \geq \max \left\{\frac{2 \sigma_{3}}{\sigma_{3}-1}, \frac{\sqrt{2} \sigma_{3}}{\sigma_{3}-2}\right\}, \quad \sigma_{1} \geq \max \left\{\frac{2 \sigma_{3}}{\sigma_{3}-1}, \frac{\sigma_{3}}{\sigma_{3}-3}\right\},
$$

then $K_{X}+B$ is free at $x$.

When $27<B^{3}<92$ one can use this to get other statements of Reider type by requiring $B \cdot C$ and $B^{2} \cdot S$ to be larger.

As an immediate consequence of Theorem 1, we obtain:

Corollary 2. Let $L$ be an ample line bundle on the smooth projective threefold $X$. Then $K_{X}+5 L$ is globally generated. If $L^{3} \geq 2$ then $K_{X}+4 L$ is free, and if $L^{3} \geq 4$ then $K_{X}+3 L$ is free.

Again the example of $\mathbb{P}^{2}$ bundles over a curve shows that there can be no numerical hypothesis on $L^{3}$ to force $K_{X}+2 L$ to be free. Note that in the setting of Fujita's conjecture, where $B=4 L$, all the inequalities in Theorem $1^{*}$ hold except for $\sigma_{1} \geq \sigma_{3} /\left(\sigma_{3}-3\right)$, which just fails. With some additional argument we overcome this difficulty and deduce the optimal statement:

Corollary 2*. If $L$ is ample, then in fact $K_{X}+4 L$ is globally generated.

Theorem $1^{*}$ is in turn a consequence of a more general result (Theorem 3.2) which also applies in the setting of Kawamata's base-point-free theorem, and we find:

Theorem 3. Let $X$ be a smooth minimal projective threefold of general type, i.e., assume that $K_{X}$ is nef and big. Then $\mathscr{O}_{X}\left(m K_{X}\right)$ is globally generated for $m \geq 7$. If $K_{X}^{3}>2$ then $\mathscr{O}_{X}\left(6 K_{X}\right)$ is free, and if $K_{X}^{3}>26$ then $\mathscr{O}_{X}\left(5 K_{X}\right)$ is free.

The theorem improves the result of Benveniste mentioned above. Again one should compare with Fujita's prediction that $\mathscr{O}_{X}\left(5 K_{X}\right)$ is always globally generated. Similarly:

Proposition 4. Let $B$ be a big and nef line bundle on a smooth projective threefold $X$ such that $K_{X}+B$ is big and nef. Then the linear system $\left|m\left(K_{X}+B\right)\right|$ is base-point free when $m \geq 7$.

One can obtain analogous statements when $K_{X}+B$ is nef but not big. 
We expect that the techniques of the present paper can also be used to obtain criteria for adjoint bundles $K_{X}+B$ to be very ample, although it seems this is not completely formal, and that some of the results can be extended to threefolds with mild singularities. We hope to return to these matters elsewhere. However, the proof of our main result is already rather long, and we thought it a kindness to both the reader and the authors to present it as is.

It may be useful to say a few words about the strategy for producing sections of $K_{X}+B$, which in many respects follows the proof of Shokurov's NonVanishing Theorem [S]. Having fixed a point $x \in X$, a lower bound on $B^{3}$ allows us to construct a divisor $D \in|n B|$ with high multiplicity at $x$. Blow up $x$, and then take an embedded resolution $f: Y \longrightarrow X$ of $D$. The argument of Kawamata-Reid-Shokurov gives a smooth divisor $E \subset Y$ with $x \in f(E)$ such that the restriction map

$$
H^{0}\left(Y, f^{*}\left(K_{X}+B\right)-N\right) \longrightarrow H^{0}\left(E,\left(f^{*}\left(K_{X}+B\right)-N\right) \mid E\right)
$$

is surjective, where $N \subset Y$ is a divisor with $N \cap f^{-1}(x)=\varnothing$. (For the experts: we ignore here the fact that in the first instance one might have to throw in a sum of exceptional components.) One thinks of $E$ as arising from the "most singular" locus of $D$. So we are reduced to showing that the group on the right in $(*)$ has a section which is nonvanishing at some point $y \in f^{-1}(x)$.

We distinguish three cases, according to whether $\operatorname{dim} f(E)=0,1$, or 2 (compare [W2]). In the first case, which would arise if the multiplicity of $D$ were much larger at $x$ than at neighboring points, $\left(f^{*}\left(K_{X}+B\right)-N\right) \mid E=\mathscr{O}_{E}$, and so we are done. If $\operatorname{dim} f(E)=1$, then there is a fibration $p: E \longrightarrow C$ of $E$ over a smooth curve $C$ mapping to $X$. Choose $Q \in C$ lying over $x \in X$, and let $Z=p^{-1}(Q)$. Roughly (but not literally) speaking, the idea is to use Kawamata-Viehweg vanishing on $E$ to show the surjectivity of

$H^{0}\left(E,\left(f^{*}\left(K_{X}+B\right)-N\right) \mid E\right) \longrightarrow H^{0}\left(Z,\left(f^{*}\left(K_{X}+B\right)-N\right) \mid Z\right)=H^{0}\left(Z, \mathscr{O}_{Z}\right)$,

so again we would be done. Finally, suppose that $\operatorname{dim} f(E)=2$, so that $E$ maps birationally to a surface $S \subset X$. To a first approximation, the group on the right in $(*)$ arises as $H^{0}\left(E, K_{E}+{ }^{r} M^{\urcorner}\right)$for some ample $\mathbb{Q}$-divisor $\mathrm{M}$ on $E$. The idea then is to prove a Reider-type theorem for such divisors and apply it to produce the required section. Unfortunately there are several technical difficulties here, and in fact we end up working on a surface between $S$ and $E$. An essential remark then is that $S$ has at worst a canonical singularity at $x$.

The paper is organized as follows. For warm-up, we start in $\S 1$ by showing how the approach of Kawamata-Reid-Shokurov leads to a proof of some of Reider's results. Our impression is that these methods are not well known outside the circle of experts, and we hope that an elementary concrete application will lead to a wider appreciation of their power. (The ideas are particularly transparent in this setting because one does not have to pass to normal crossings.) In $\S 2$ we prove a Reider-type theorem for $\mathbb{Q}$-divisors on a surface, which may be of some independent interest. The statement of the main Theorem 3.2 appears in $\S 3$, where we also introduce the set-up of the proof. The argument is completed in $\S \S 4$ and 5. In $\S 6$ we indicate the modifications necessary to prove Corollary $2^{*}$. 
We have benefitted from discussions with J.-P. Demailly, J. Li, M. Reid, G. $\mathrm{Xu}$, and especially $\mathrm{G}$. Fernandez del Busto. The philosophy of how KawamataViehweg vanishing can apply to give sections of $K_{X}+B$ was first explained to us by $\mathrm{H}$. Esnault and $\mathrm{E}$. Viehweg in connection with a lecture by Siu, and we thank them for getting us started. We are particularly grateful to Y.-T. Siu for his kindness and patience in explaining to us the analytic viewpoint on these matters and the work of Demailly. Finally, we thank J. Kollár for commenting on an early draft of this paper and for showing us preliminary versions of [Kol2].

\section{Notation and Conventions}

(0.1) We work throughout over the complex numbers $\mathbb{C}$.

(0.2) If $X$ is a smooth variety of dimension $n$, we denote by $K_{X}$ a canonical divisor on $X$. We say that a divisor (or line bundle or linear series) B is free at a point $x \in X$ if $\mathscr{O}_{X}(B)$ has a section which is nonvanishing at $x$. We say that $B$ is free (or base-point free or globally generated) if $\mathscr{O}_{X}(B)$ is free at every point of $X$. Recall that $B$ is numerically effective or nef if $B \cdot C \geq 0$ for all curves $C \subset X$. If $B$ is nef, it is in addition big if $B^{n}>0$. Note that if $f: Y \longrightarrow X$ is a generically finite surjective map of projective varieties, and if $B$ is a big and nef divisor on $X$, then $f^{*} B$ is big and nef on $Y$.

$(0.3)$ We review the customary notation concerning $\mathbb{Q}$-divisors. Given a rational number $a$, the round-up ${ }^{\top} a^{7}$ of $a$ is the least integer $\geq a$, and the fractional part $\{a\}$ is $a-[a]$, where as usual $[a]$ is the integer part or round-down of $a$. Thus, for instance, $\left.{ }^{\ulcorner}-\frac{1}{2}\right\urcorner=\left[\frac{1}{3}\right]=\{2\}=0$. A $\mathbb{Q}$-divisor on an algebraic variety $X$ is a Weil-divisor $D=\sum a_{i} \cdot D_{i}$ with $\mathbb{Q}$-coefficients. The group of all such is $\operatorname{Div}_{\mathbb{Q}}(X)$, and given a prime divisor $E$ on $X, \operatorname{ord}_{E}(D)$ denotes the coefficient of $E$ in $D$. Assuming that the $D_{i}$ are distinct prime divisors, the round-up of $D$ is $\left.{ }^{\ulcorner} D^{\urcorner}=\sum{ }^{r} a_{i}\right\urcorner \cdot D_{i}$. The fractional part $\{D\}$ and the integral part $[D]$ of $D$ are defined similarly. If all the $D_{i}$ are Cartier (or $\mathbb{Q}$-Cartier, i.e., some multiple of each is Cartier), there is the usual notion of numerical equivalence, which we denote by $\equiv$, and it makes perfectly good sense to say that $D$ is ample, or nef and big. We refer to [CKM] or [KMM] for details.

(0.4) The basic tool underlying the approach of Kawamata-Reid-Shokurov is the Kawamata-Viehweg Vanishing Theorem:

Let $X$ be a smooth complex projective variety, and let $M$ be a big and nef $\mathbb{Q}$-divisor on $X$. Assume that the fractional part $\{M\}$ of $M$ is supported on a divisor with global normal crossings. Then $\left.H^{i}\left(X, \mathscr{O}_{X}\left(K_{X}+{ }^{r} M\right\urcorner\right)\right)=0$ for $i>$ 0 .

Note that, since rounding does not in general commute with linear equivalence, it is important here that the fractional part of $M$ be defined on the level of divisors. However, we will often tacitly identify two $\mathbb{Q}$-divisors whose fractional parts coincide and whose integral parts are linearly equivalent. By the same token, we will deal with objects of the form $L(D)$, where $L$ is a line bundle (defined up to isomorphism) and $D \in \operatorname{Div}_{\mathbb{Q}}(X)$; we refer to these also as $\mathbb{Q}$-divisors. Note that, if $L(D)$ is such a $\mathbb{Q}$-divisor and $E \subset X$ is any prime divisor, then the restriction $L(D) \mid E$ makes sense as a $\mathbb{Q}$-divisor provided that 
$E$ does not appear in the fractional part of $D$, i.e., $\operatorname{ord}_{E}(\{D\})=0$. If, in addition, $E$ and all the components of $\{D\}$ form a normal crossing divisor, then rounding commutes with restriction. However, when $\operatorname{ord}_{E}(\{D\}) \neq 0$, the restriction $L(D) \mid E$ is only defined as a linear equivalence class, i.e. as an element in $\operatorname{Pic}_{\mathbb{Q}}(X)$. (As a practical matter, one restricts in the latter case for numerical calculations but not as input to Vanishing.) Besides [CKM] and [KMM], we recommend the articles [Kol1, W1] of Kollár and Wilson, as well as the notes [EV] of Esnault and Viehweg, as good introductions to this circle of ideas.

\section{KAWAMATA-VIEHWEg VANISHING AND REIDER'S THEOREM}

This section is intended for the benefit of the nonexpert reader. We show how the approach of Kawamata-Reid-Shokurov leads to a simple proof of some of the statements from [Rdr]. The result we prove is actually a special case of Theorem 2.3 below, but with the technicalities stripped away. This renders the arguments particularly transparent, and at the risk of repetition we include them here in the hope of making subsequent sections (as well as the general techniques of Kawamata-Reid-Shokurov) more accessible to the uninitiated.

One of the pleasant features of working on surfaces is that vanishing for $\mathbb{Q}$-divisors holds without any normal crossing hypotheses:

1.1. Lemma [Sak1]. Let $S$ be a smooth projective surface, and let $M$ be any big and nef $\mathbb{Q}$-divisor on $S$. Then

$$
H^{i}\left(S, \mathscr{O}_{S}\left(K_{X}+{ }^{r} M^{\urcorner}\right)\right)=0 \text { for } i>0 .
$$

Proof. By a succession of blowings-up at points, we construct a map $\phi$ : $S_{1} \longrightarrow S$ such that $\left\{\phi^{*} M\right\}$ is supported on a normal crossing divisor. Since $\phi^{*} M$ is still nef and big, Kawamata-Viehweg vanishing (0.4) gives

$$
H^{i}\left(S_{1}, \mathscr{O}_{S_{1}}\left(K_{S_{1}}+{ }^{\ulcorner} \phi^{*} M^{\urcorner}\right)\right)=0 \text { for } i>0 .
$$

Working our way down from $S_{1}$, it is therefore enough to prove:

(1.2) Let $f: Y \longrightarrow X$ be the blowing-up of a smooth surface at a point $x \in X$, and let $M$ be a $\mathbb{Q}$-divisor on $X$. If $\left.H^{i}\left(Y, \mathscr{O}_{Y}\left(K_{Y}+{ }^{r} f^{*}(M)\right\urcorner\right)\right)=0$ for some $i>0$, then $\left.H^{i}\left(X, \mathscr{O}_{X}\left(K_{X}+{ }^{\ulcorner} M\right\urcorner\right)\right)=0$.

For (1.2), write $M=\ulcorner M\urcorner-\sum a_{j} D_{j}\left(0<a_{j}<1\right)$, where the $D_{j} \subset X$ are distinct prime divisors. Let $E \subset Y$ be the exceptional divisor over $x$, and let $D_{j}^{\prime} \subset Y$ be the proper transform of $D_{j}$. Then $f^{*} D_{j}=D_{j}^{\prime}+q_{j} E$ for some $q_{j} \geq 0$, whence

$$
f^{*} M=f^{*}\ulcorner M\urcorner-\sum a_{j} D_{j}^{\prime}-q E \quad(q \geq 0) .
$$

Since $K_{Y}=f^{*} K_{X}+E$, we find that

$$
\left.\left.K_{Y}+{ }^{r} f^{*} M\right\urcorner=f^{*}\left(K_{X}+{ }^{\ulcorner} M\right\urcorner\right)-p E,
$$

where $p=[q]-1 \geq-1$. Therefore,

$$
\begin{aligned}
f_{*}\left(\mathscr{O}_{Y}\left(K_{Y}+{ }^{r} f^{*} M^{\urcorner}\right)\right) & =\mathscr{O}_{X}\left(K_{X}+{ }^{r} M^{\urcorner}\right) \otimes \mathfrak{m}_{x}^{p}, \\
R^{k} f_{*}\left(\mathscr{O}_{Y}\left(K_{Y}+{ }^{r} f^{*} M^{\urcorner}\right)\right) & =0 \quad \text { for } k>0,
\end{aligned}
$$


where $\mathfrak{m}_{x}$ is the ideal sheaf of $x$, with the convention that $\mathfrak{m}_{x}^{p}=\mathscr{O}_{X}$ when $p=-1$. Hence,

$$
\left.\left.H^{i}\left(Y, \mathscr{O}_{Y}\left(K_{Y}+{ }^{r} f^{*} M\right\urcorner\right)\right)=H^{i}\left(X, \mathscr{O}_{X}\left(K_{X}+{ }^{r} M\right\urcorner\right) \otimes \mathfrak{m}_{x}^{p}\right)
$$

for all $i$. But $\left.H^{i}\left(X, \mathscr{O}_{X}\left(K_{X}+{ }^{r} M\right\urcorner\right) \otimes \mathscr{O}_{X} / \mathfrak{m}_{x}^{p}\right)=0$ when $i>0$ since the sheaf in question is supported on a point, and therefore (1.2) follows from $(*)$.

1.3. Remark. In general, suppose that $M$ is any big and nef $\mathbb{Q}$-divisor on a smooth complex projective variety $X$. Then there is an ideal sheaf $\mathscr{J} \subset \mathscr{O}_{X}$, with $\mathscr{O}_{X} / \mathscr{J}$ supported on a subset of codimension $\geq 2$, such that

$$
H^{i}\left(X, \mathscr{O}_{X}\left(K_{X}+{ }^{\ulcorner} M^{\urcorner}\right) \otimes \mathscr{J}\right)=0 \text { for } i>0 .
$$

Demailly informs us that one can view the sheaf $\mathscr{J}$ as one of the multiplier ideals that appear in the work of Nadel and in [Dem].

The remainder of this section is devoted to the following special case of Reider's theorem [Rdr]:

1.4. Proposition. Let $B$ be a big and nef divisor on a smooth projective surface $S$, and let $x \in S$ be a fixed point. Assume that:

(i) $B^{2} \geq 5$.

(ii) $B \cdot C \geq 2 \quad \forall$ curves $C \subset S$ with $x \in C$.

Then $\mathscr{O}_{S}\left(K_{S}+B\right)$ has a section which is nonvanishing at $x$.

Reider actually shows that if $x \in S$ is a base point for $K_{S}+B$, then there is a curve $C \subset S$ through $x$ with $B \cdot C=1$ and $C^{2}=0$ or $B \cdot C=0$ and $C^{2}=-1$. From the present point of view, it would take additional work to rule out the possibility $C^{2} \ll 0$.

The idea of the proof is this: we use (i) to construct a divisor $D \in|n B|(n \gg$ 0 ) with suitably high multiplicity at $x$. If $D$ is irreducible - or more generally if $2 \cdot$ mult $_{y}(D)<$ mult $_{x}(D)$ for $y$ near $x$ - then we have a vanishing which directly gives the required section. (See [SS, (7.56)] for a "classical" argument of this type and [EV, (7.5)-(7.8)] for an analogous fact in all dimensions.) In the alternative case, there is a component $D_{0}$ of $D$ with high multiplicity. The approach of Kawamata-Reid-Shokurov is to reduce the question to producing a nonvanishing section on $D_{0}$, which we attack using hypothesis (ii).

1.5. Proof of (1.4). To begin with, note that for $n \gg 0$ there exists a divisor

$$
D \in|n B| \quad \text { with } \quad q=\text { mult }_{x}(D) \geq 2 n+1 .
$$

In fact, by Riemann Roch and $(\mathrm{i}), h^{0}\left(X, \mathscr{O}_{S}(n B)\right)$ grows like $n^{2} B^{2} / 2 \geq 5 n^{2} / 2$, whereas it is $\left(\begin{array}{c}2 n+2 \\ 2\end{array}\right) \sim 4 n^{2} / 2$ conditions to impose a point of multiplicity $\geq$ $2 n+1$ at $x$. Choose such a divisor $D$, and write

$$
D=\sum d_{i} D_{i}+F
$$

where the $D_{i}$ are the prime components of $D$ passing through $x$ and $F$ is the effective divisor comprised of those components of $D$ disjoint from $x$. 
(1.6) Suppose first that $q>2 d_{i}$ for all i. Then let $f: Y \longrightarrow S$ be the blowing-up of $x$, let $E \subset Y$ be the exceptional divisor, and let $D_{i}^{\prime} \subset Y$ be the proper transform of $D_{i}$. Thus $f^{*} D=\sum d_{i} D_{i}^{\prime}+q E+f^{*} F$. Consider then the Q-divisor

$$
M=f^{*} B-\frac{2}{q} f^{*} D=f^{*} B-2 E-\sum \frac{2 d_{i}}{q} D_{i}^{\prime}-\frac{2}{q} f^{*} F
$$

on $\mathrm{Y}$. Now $2 d_{i} / q<1$, so $\ulcorner M\urcorner=f^{*} B-2 E-N$, where $N=\left[\frac{2}{q} f^{*} F\right]$ is an effective divisor supported away from $E$. Thus

$$
K_{Y}+{ }^{r} M^{\urcorner}=f^{*}\left(K_{S}+B\right)-E-N .
$$

On the other hand, $M \equiv\left(1-\frac{2 n}{q}\right) f^{*} B$, and by construction $1-\frac{2 n}{q}>0$. Therefore, $M$ is big and nef, and vanishing (1.1) yields

$$
H^{1}\left(Y, \mathscr{O}_{Y}\left(f^{*}\left(K_{S}+B\right)-E-N\right)\right)=H^{1}\left(S, \mathscr{O}_{S}\left(K_{S}+B-N^{\prime}\right) \otimes \mathfrak{m}_{x}\right)=0,
$$

where $N^{\prime}=f_{*} N \subset S$ is a divisor supported away from $x$ and $\mathfrak{m}_{x}$ is the ideal sheaf of $x$. It follows that there is a section $t \in H^{0}\left(S, \mathscr{O}_{S}\left(K_{S}+B-N^{\prime}\right)\right)$ such that $t(x) \neq 0$. Since $x \notin \operatorname{Supp}\left(N^{\prime}\right)$, this yields the required section of $\mathscr{O}_{S}\left(K_{S}+B\right)$.

(1.7) Suppose next that $q<2 d_{i}$ for some $i$. Note from (1.5.1) that

$$
q=\sum d_{i} \cdot \operatorname{mult}_{x}\left(D_{i}\right),
$$

so in the first place $q \geq d_{i}$ for all $i$. It follows that there is a unique component $D_{i}$, say $D_{0}$, of maximal multiplicity $d_{0}>\frac{q}{2}$. Furthermore, we see that $D_{0}$ is smooth at $x$. Consider now on $S$ the $\mathbb{Q}$-divisor $M=B-D / d_{0}$. As above $M \equiv\left(1-n / d_{0}\right) B$ is big and nef, and

$$
\left.K_{S}+{ }^{r} M\right\urcorner=K_{S}+B-D_{0}-N
$$

for some effective divisor $N \subset S$ supported away from $x$. It follows from the vanishing (1.1) that $H^{1}\left(S, \mathscr{O}_{S}\left(K_{S}+B-D_{0}-N\right)\right)=0$. Therefore, the restriction map

$$
H^{0}\left(S, \mathscr{O}_{S}\left(K_{S}+B-N\right)\right) \longrightarrow H^{0}\left(D_{0}, \mathscr{O}_{S}\left(K_{S}+B-N\right) \mid D_{0}\right)
$$

is surjective. To complete the proof it is enough to show that the group on the right in (1.7.2) has a section $\bar{t}$ which does not vanish at at $x$, for then $\bar{t}$ lifts to $t \in H^{0}\left(S, \mathscr{O}_{S}\left(K_{S}+B-N\right)\right)$ and we conclude as above.

(1.8) The existence of the required section $\bar{t}$ will in turn follow if we verify

$$
\left(B-D_{0}-N\right) \cdot D_{0}>1 \text {. }
$$

For then, since $\left(B-D_{0}-N\right) \cdot D_{0}$ is in any event an integer, the sheaf on the right in (1.7.2) is of the form $\omega_{D_{0}} \otimes L$, where $L$ is a line bundle of degree $\geq 2$, and hence is free at $x$. As for (1.8.1) note that $\ulcorner M\urcorner-M=\sum_{i \geq 1} d_{i} D_{i} / d_{0}+\Delta$, 
where $\left.\Delta={ }^{r} F / d_{0}\right\urcorner-F / d_{0}$ is an effective divisor which meets $D_{0}$ properly. Thus

$$
B-D_{0}-N=\ulcorner M\urcorner \equiv M+\sum_{i \geq 1} \frac{d_{i}}{d_{0}} D_{i}+\Delta .
$$

But $B \cdot D_{0} \geq 2$ by assumption, $D_{i} \cdot D_{0} \geq \operatorname{mult}_{x}\left(D_{i}\right)$ for $i \geq 1$, and $M \equiv$ $\left(1-n / d_{0}\right) B$. Then (1.8.1) follows with a little calculation using (1.7.1).

(1.9) It remains to treat the borderline possibility that $q=2 d_{i}$ for some $i$. The general approach of Kawamata-Reid-Shokurov would be to perturb slightly the coeffiecients of the components occurring in $M$. But in the present setting, it is easier to argue as in (1.6) using the Norimatsu-type Lemma 2.4 below to remove the offending $D_{i}$. We leave details to the reader.

By an analogous argument, we will prove in the next section (a generalization of) the following

1.10. Variant. In the situation of (1.4), suppose that $M$ is a nef $\mathbb{Q}$-divisor on $S$ such that

$$
M \cdot M>4, \quad \text { and } \quad M \cdot C \geq 2 \quad \forall \text { curves } C \ni x .
$$

Then $\left.K_{S}+{ }^{r} M\right\urcorner$ is free at $x$.

It would be interesting to know whether this has any applications to surfaces.

\section{A THEOREM OF REIDER TYPE FOR $\mathbb{Q}$-DIVISORS ON A SURFACE}

In this section we formulate and prove the Reider-type theorem for $\mathbb{Q}$ divisors that underlies our work on threefolds. In the interests of future reference, we give the result in slightly more generality than will be needed in the sequel.

(2.1) We start with some remarks about the multiplicity of a fractional divisor at a point. Let $(S, x)$ be a normal surface germ. We assume that $x$ is either a smooth point or a rational double point (RDP) of $S$. Let $\phi: S^{\prime} \rightarrow S$ be the blowing up of $S$ at $x$ if $x$ is a smooth point, or the minimal resolution of the germ $(S, x)$ if $x$ is a RDP. Denote by $Z$ the exceptional divisor over $x$ in the first case, or the fundamental cycle in the RDP case (cf. [BPV, III.2 ] for the basic facts about RDP's). Now suppose that $\Delta$ is an effective $\mathbb{Q}$-divisor on $S$. Then we define the multiplicity mult $_{x}(\Delta)$ of $\Delta$ at $x$ to be the largest rational number $\mu$ such that $\mu Z \leq \phi^{*} \Delta$. (The pull-back makes sense thanks to the fact that $\Delta$ is automatically $\mathbb{Q}$-Cartier near $x$.) At least when $x$ is a smooth point, this is the evident notion: if we write $\Delta=\sum n_{i} \Delta_{i}$ as a sum of prime divisors, then mult $_{x}(\Delta)=\sum n_{i} \cdot$ mult $_{x}\left(\Delta_{i}\right)$. Note that, if $f: S \longrightarrow M$ is a map from $S$ to a smooth variety and $D=\sum r_{i} D_{i}$ is a $\mathbb{Q}$-divisor on $M$ whose support meets $f(S)$ properly, then

$$
\text { mult }_{x}\left(f^{*} D\right) \geq \text { mult }_{f(x)}(D)=_{\text {def }} \sum r_{i} \cdot \text { mult }_{f(x)}\left(D_{i}\right)
$$

[Proof. By linearity it is enough to check this when $D$ is Cartier, and then it follows from the fact that, if $g \in \mathfrak{m}_{x}^{\ell}$ is a local equation for $f^{*}(D)$, then $\ell Z \leq \operatorname{div}\left(\phi^{*} g\right)$.] 
2.2. Notation and assumptions. We consider a surjective map of irreducible projective surfaces

$$
h: S_{1} \longrightarrow S_{0}
$$

and points $x_{1} \in S_{1}, x_{0} \in S_{0}$ such that $h\left(x_{1}\right)=x_{0}$. Assume that $S_{1}$ is smooth except perhaps for finitely many rational double points along $h^{-1}\left(x_{0}\right)$ (so that in particular, $S_{1}$ is Gorenstein and $\mathbb{Q}$-factorial). Let $M$ be a big and nef $\mathbb{Q}$ divisor on $S_{1}$ with the property that ${ }^{r} M^{\urcorner}$is Cartier. We fix positive rational numbers $\beta_{1}, \beta_{2}$ such that

(2.2.1) $M \cdot M>\left(\beta_{2}\right)^{2}$,

(2.2.2) $\quad M \cdot \Gamma>\beta_{1} \quad \forall$ curves $\Gamma \subset S_{1}$ s.t. $h(\Gamma)$ is a curve through $x_{0}$.

Let $\left.\Delta={ }^{r} M\right\urcorner-M$ be the difference between $M$ and its round-up, and fix a rational number $0 \leq \mu \leq \operatorname{mult}_{x_{1}}(\Delta)$.

2.3. Theorem. In the situation of (2.2), suppose that $\left.\mathscr{O}_{S_{1}}\left(K_{S_{1}}+{ }^{r} M\right\urcorner\right) \mid \Gamma \cong \mathscr{O}_{\Gamma}$ for every reduced (but possibly reducible) curve $\Gamma \subset S_{1}$ such that $h(\Gamma)=x_{0}$. Assume also that

$(2.3 .1)_{\text {smooth }}$

$$
\beta_{2} \geq 2-\mu \quad \text { and } \quad \beta_{1}\left(1-\frac{2-\mu}{\beta_{2}}\right) \geq 1
$$

when $x_{1}$ is a smooth point of $S_{1}$, or

$$
\beta_{2} \geq(1-\mu) \sqrt{2} \text { and } \beta_{1}\left(1-\frac{1-\mu}{\beta_{2}} \sqrt{2}\right) \geq 1
$$

when $x_{1}$ is an RDP of $S_{1}$. Then $\left.\mathscr{O}_{S_{1}}\left(K_{S_{1}}+{ }^{r} M\right\urcorner\right)$ has a section which is nonvanishing at some point $x \in h^{-1}\left(x_{0}\right)$. If, moreover, $h^{-1}\left(x_{0}\right)=\left\{x_{1}\right\}$, then the same conclusion holds (with $x=x_{1}$ ) also under the hypotheses:

$$
\beta_{2} \geq 2-\mu \text { and } \beta_{1} \geq 2-\mu
$$

when $x_{1}$ is smooth, or

$$
\beta_{2} \geq(1-\mu) \sqrt{2} \text { and } \beta_{1} \geq(1-\mu)
$$

when $x_{1}$ is a rational double point.

In order to avoid a somewhat unpleasant "tie-breaking" argument, we will base the proof on Kawamata-Viehweg vanishing in the form of a Norimatsutype statement:

2.4. Lemma. Let $R$ be a big and nef $\mathbb{Q}$-divisor on a smooth surface $S$. Let $E_{1}, \ldots, E_{k}$ be distinct irreducible curves on $S$ which do not appear in the fractional part of $R$, i.e., with $\operatorname{ord}_{E_{i}}(\{R\})=0$. Assume that $R \cdot E_{i}>0$ for all $1 \leq i \leq k$. Then

$$
H^{i}\left(S, \mathscr{O}_{S}\left(K_{S}+{ }^{r} R^{\urcorner}+E_{1}+\cdots+E_{k}\right)\right)=0 \text { for } i>0 .
$$

Proof. When $k=0$, this is the Kawamata-Viehweg Vanishing Theorem (1.1). In general, one argues by induction on $k$. One has $\operatorname{deg}\left(\left\ulcorner R^{\urcorner} \cdot E\right) \geq \operatorname{deg}\left(R \cdot E_{i}\right)>0\right.$ 
thanks to the hypothesis that $\operatorname{ord}_{E_{i}}(\{R\})=0$. The lemma then follows by taking cohomology from the exact sequence

$$
\begin{aligned}
0 & \left.\left.\longrightarrow \mathscr{O}_{S}\left(K_{S}+{ }^{r} R\right\urcorner+E_{1}+\cdots+E_{i-1}\right) \longrightarrow \mathscr{O}_{S}\left(K_{S}+{ }^{r} R\right\urcorner+E_{1}+\cdots+E_{i}\right) \\
& \longrightarrow \mathscr{O}_{E_{i}}\left(K_{E_{i}}+{ }^{r} R^{\urcorner}+E_{1}+\cdots+E_{i-1}\right) \longrightarrow 0 .
\end{aligned}
$$

Proof of Theorem 2.3. We will assume that $x_{1}$ is a RDP of $S_{1}$. The argument when $x_{1}$ is a smooth point is similar [and the required changes will be indicated within brackets].

(2.5) For all sufficiently large integers $n$ such that $n M$ is Cartier, (2.2.1) and Riemann-Roch imply that $h^{0}\left(S_{1}, \mathscr{O}_{S_{1}}(n M)\right)>n^{2}\left(\beta_{2}+\epsilon\right)^{2} / 2$ for suitable $\epsilon>0$. On the other hand, since $x_{1}$ is a double point, $h^{0}\left(S_{1}, \mathscr{O}_{S_{1}} / \mathfrak{m}_{x_{1}}^{\ell}\right)=2 \ell^{2} / 2+$ (lower order terms in $\ell$ ), where $\mathfrak{m}_{x_{1}}$ is the ideal sheaf of $x_{1}$ in $S_{1}$. Therefore there exists an integer $n \gg 0$ with $n M$ Cartier, plus a nonzero section $s \in$ $H^{0}\left(S_{1}, \mathscr{O}_{S_{1}}(n M) \otimes \mathfrak{m}_{x_{1}}^{q_{1}}\right)$ for some $q_{1}>n \beta_{2} / \sqrt{2}$. Let $D=\operatorname{div}(s) \subset S_{1}$ be the corresponding divisor. [When $x_{1}$ is a smooth point, one takes $q_{1}>n \beta_{2}$.]

(2.6) Let $\phi: S \longrightarrow S_{1}$ be the minimal resolution of $S_{1}$, and let $g=h \circ \phi$ : $S \longrightarrow S_{0}$ be the composition of $\phi$ with $h$. Thus $K_{S}=\phi^{*} K_{S_{1}}$. Denote by $Z$ the fundamental cycle in $S$ lying over $x_{1}$. [When $x_{1}$ is smooth, we construct $S$ by first blowing up $x_{1}$ and then taking the minimal resolution of the resulting surface. In this case $Z$ is the exceptional divisor over $x_{1}$, and we have $K_{S}=\phi^{*} K_{S_{1}}+Z$.] For suitable irreducible divisors $\left\{E_{j}\right\}$ on $S$ and numbers $r_{j} \in \mathbb{N}, a_{j} \in \mathbb{Q}$, we may write

$$
\begin{gathered}
\phi^{*} D=\sum r_{j} \cdot E_{j}, \\
\phi^{*} \Delta=\phi^{*}(\ulcorner M\urcorner-M)=\sum a_{j} \cdot E_{j} .
\end{gathered}
$$

Note that if $E_{i}$ is not $\phi$-exceptional, then $0 \leq a_{i}<1$. Furthermore, if $Z^{\prime}$ is one of the components of $Z$, with corresponding coefficients $a^{\prime}$ and $r^{\prime}$, then $r^{\prime} \geq q_{1}$ and $a^{\prime} \geq$ mult $_{x_{1}}(\Delta) \geq \mu$.

(2.7) Suppose first that $a_{j} \geq 1$ for some $j$. Then ${ }^{\ulcorner}\left(\phi^{*}\ulcorner M\urcorner-\sum a_{j} E_{j}\right)^{\urcorner}=$ $\phi^{*} r M^{\urcorner}-W$, where $W \neq 0$ is a positive linear combination of $\phi$-exceptional divisors (lying over $x_{0}$ ). On the other hand, $\left.\phi^{*} r M\right\urcorner-\sum a_{j} E_{j} \equiv \phi^{*}(M)$ is nef and big, so by vanishing we conclude that $H^{1}\left(S, \mathscr{O}_{S}\left(K_{S}+\phi^{*}\ulcorner M\urcorner-W\right)\right)=0$. Hence, $H^{1}\left(S_{1}, \phi_{*} \mathscr{O}_{S}\left(K_{S}+\phi^{*}\ulcorner M\urcorner-W\right)\right)=0$. But

$$
\left.\phi_{*} \mathscr{O}_{S}\left(K_{S}+\phi^{*}\ulcorner M\urcorner-W\right)=\mathscr{O}_{S_{1}}\left(K_{S_{1}}+{ }^{r} M\right\urcorner\right) \otimes \mathscr{J}_{T},
$$

where $T \subset S_{1}$ is a nonempty finite scheme supported in $h^{-1}\left(x_{0}\right)$. We conclude that $\mathscr{O}_{S_{1}}\left(K_{S_{1}}+{ }^{r} M^{\urcorner}\right)$has a section which is nonvanishing on $\operatorname{supp}(T)$, as required. [When $x_{1}$ is smooth and $E_{0}=Z$ is the exceptional divisor over it, this argument works when $a_{j} \geq 1$ for $j \neq 0$ or $a_{0} \geq 2$.] 
(2.8) So we may assume henceforth that all $a_{j}<1$ and, in particular, that $\mu<1$. Put

$$
c=\min \left\{\frac{1-a_{j}}{r_{j}} \mid g\left(E_{j}\right) \ni x_{0}, r_{j} \neq 0\right\} .
$$

Reindexing if necessary, let $E_{1}, \ldots, E_{\ell}$ be the minimizing components where the minimum is achieved: thus $g\left(E_{i}\right) \ni x_{0}$, and $\left(1-a_{i}\right) / r_{i}=c$ for $1 \leq i \leq \ell$. All told, we have arranged things so that $a_{i}+c r_{i} \leq 1$ for any $E_{i}$ with $x_{0} \in$ $g\left(E_{i}\right)$, and equality holds if and only if $1 \leq i \leq \ell$. Let us also choose indices in such a way that $E_{1}, \ldots, E_{k}$ map to curves in $S_{0}$, whereas $g\left(E_{k+1}\right)=\cdots=$ $g\left(E_{\ell}\right)=x_{0}$. (If all the minimizing components are $g$-exceptional, take $k=0$.) Finally, put

$$
E^{\prime}=E_{1}+\cdots+E_{k}, \quad E^{\prime \prime}=E_{k+1}+\cdots+E_{\ell} .
$$

[When $x_{1}$ is a smooth point and $E_{0}=Z$ is the exceptional divisor lying over it, we assume that $a_{0}<2$, and the corresponding coefficient in the definition of $c$ is $\left(2-a_{0}\right) / r_{0}$. There are then three possibilities: $\left[a_{0}+c r_{0}\right]=2,1$, or 0 . We consider $E_{0}$ to be minimizing (and hence appearing in $E^{\prime \prime}$ ) in the first case, but not otherwise. The third case-when $\left[a_{0}+c r_{0}\right]=0$-will require some slight additional arguments. However, observe this can only occur if none of the minimizing components meet $Z$, for if $x_{1} \in \phi\left(E_{i}\right)$ for some $1 \leq i \leq \ell$, then $a_{i} \leq a_{0}$ and $r_{i} \leq r_{0}$, whence $a_{0}+c r_{0} \geq 1$.]

(2.9). By examining the coefficients attached to the components of $Z$, one finds that

$$
1-c n>1-\frac{1-\mu}{\beta_{2}} \cdot \sqrt{2} \geq 0 .
$$

Consider now the $\mathbb{Q}$-divisor $R={ }_{\text {def }} \phi^{*}\ulcorner M\urcorner-\sum\left(a_{j}+c r_{j}\right) E_{j}$. Then in the first place

$$
R \equiv \phi^{*}(M-c D) \equiv(1-c n) \phi^{*}(M),
$$

so $R$ is big and nef. Furthermore,

$$
{ }^{\ulcorner} R^{\urcorner}=\phi^{*}\ulcorner M\urcorner-E^{\prime}-E^{\prime \prime}-N,
$$

where $N$ is an effective divisor on $\mathrm{S}$ such that $\operatorname{supp}(N) \cap g^{-1}\left(x_{0}\right)=\varnothing$. [When $x_{1}$ is smooth, $(2.9 .1)$ is replaced by $1-c n>1-(2-\mu) / \beta_{2} \geq 0$. Referring to the three possibilities discussed at the end of (2.8), in the first two we have $\left.{ }^{r} R^{\urcorner}=\phi^{*} r M\right\urcorner-Z-E^{\prime}-E^{\prime \prime}-N$. When $\left[a_{0}+c r_{0}\right]=0,(2.9 .3)$ holds as stated, and we return to this case below.]

(2.10) Suppose now that $E^{\prime \prime} \neq 0$ or, in other words, that at least one of the minimizing components is $g$-exceptional. For each component $E_{i}$ of $E^{\prime}$ we have $R \cdot E_{i}=(1-c n) M \cdot \phi_{*}\left(E_{i}\right) \geq(1-c n) \beta_{1}>0$. Consequently

$$
H^{1}\left(S, \mathscr{O}_{S}\left(K_{S}+\phi^{*}\ulcorner M\urcorner-E^{\prime \prime}-N\right)\right)=0
$$

by Lemma 2.4 , and hence the restriction map

$$
H^{0}\left(S, \mathscr{O}_{S}\left(K_{S}+\phi^{*}\ulcorner M\urcorner-N\right)\right) \longrightarrow H^{0}\left(E^{\prime \prime}, \mathscr{O}_{S}\left(K_{S}+\phi^{*}\ulcorner M\urcorner-N\right) \mid E^{\prime \prime}\right)
$$


is surjective. On the other hand, considering $E^{\prime \prime}$ as a reduced curve on $S$, note that $\mathscr{O}_{E^{\prime \prime}}(N) \cong \mathscr{O}_{E^{\prime \prime}}$ since $E^{\prime \prime} \cap \operatorname{supp}(N)=\varnothing$ and observe that $\Gamma=_{\text {def }} \phi\left(E^{\prime \prime}\right)$ maps under $h$ to $x_{0}$. It follows from the first hypothesis of the theorem that

$$
\left.\mathscr{O}_{S}\left(K_{S}+\phi^{*}\ulcorner M\urcorner-N\right) \mid E^{\prime \prime} \cong \phi^{*}\left(\mathscr{O}_{S_{1}}\left(K_{S_{1}}+{ }^{\ulcorner} M\right\urcorner\right)\right) \mid E^{\prime \prime}=\mathscr{O}_{E^{\prime \prime}} .
$$

By the surjectivity $(*)$, there thus exists a section

$$
t^{\prime} \in H^{0}\left(S, \mathscr{O}_{S}\left(K_{S}+\phi^{*}\ulcorner M\urcorner-N\right)\right)
$$

which is nowhere vanishing on $E^{\prime \prime}$. Thanks again to the fact that $E^{\prime \prime}$ is disjoint from the support of $N$, this implies the existence of a section

$$
\left.t \in H^{0}\left(S, \mathscr{O}_{S}\left(K_{S}+\phi^{*}\ulcorner M\urcorner\right)\right)=\phi^{*} H^{0}\left(S_{1}, \mathscr{O}_{S_{1}}\left(K_{S_{1}}+{ }^{\ulcorner} M\right\urcorner\right)\right)
$$

which is nowhere vanishing on $E^{\prime \prime}$, and the assertion of the theorem follows in the case at hand. [When $x_{1}$ is smooth, recall that $K_{S}=\phi^{*}\left(K_{S_{1}}\right)+Z$. In the first two cases discussed in (2.8) - where ${ }^{\ulcorner} R^{\urcorner}=\phi^{*}\ulcorner M\urcorner-Z-E^{\prime}-E^{\prime \prime}-N-$ the argument just given works without any essential changes. But when $\left[a_{0}+c r_{0}\right]=$ 0 , some amplifications may be in order. In this case (2.9.3) and the surjectivity $(*)$ hold as stated, and this creates a potential difficulty in passing from $K_{S}$ to $K_{S_{1}}$. However, recall from (2.8) that $E^{\prime \prime} \cap Z=\varnothing$ in the case at hand, and therefore the isomorphisms in (2.10.1) remain valid. So we conclude as above the existence of a section $t \in H^{0}\left(S, \mathscr{O}_{S}\left(K_{S}+\phi^{*}\ulcorner M\urcorner\right)\right)$ nowhere vanishing on $E^{\prime \prime}$. But

$\left.H^{0}\left(S_{1}, \mathscr{O}_{S_{1}}\left(K_{S_{1}}+{ }^{\ulcorner} M^{\urcorner}\right)\right)=H^{0}\left(S, \phi^{*} \mathscr{O}_{S_{1}}\left(K_{S_{1}}+{ }^{\ulcorner} M^{\urcorner}\right\urcorner\right)\right) \cong H^{0}\left(S, \mathscr{O}_{S}\left(K_{S}+\phi^{*}\ulcorner M\urcorner\right)\right)$, where the second isomorphism is given by multiplication by $Z$ (cf. (3.10)). So we do in fact get the required section of $\mathscr{O}_{S_{1}}\left(K_{S_{1}}+{ }^{r} M^{\urcorner}\right)$.]

(2.11) It remains only to treat the case when $E^{\prime \prime}=0$, i.e., when all the minimizing components map to curves in $S_{0}$. To this end, we argue first that it is enough to establish the inequality

$$
\ulcorner R\urcorner \cdot E_{1}>1 \text {. }
$$

In fact, suppose for the time being that $(2.11 .1)$ is known. Applying Lemma 2.4 to the divisor $E_{2}+\cdots+E_{k}$, we find as above that the restriction

$$
H^{0}\left(S, \mathscr{O}_{S}\left(K_{S}+\phi^{*}\ulcorner M\urcorner-N\right)\right) \longrightarrow H^{0}\left(E_{1}, \mathscr{O}_{S}\left(K_{S}+\phi^{*}\ulcorner M\urcorner-N\right) \mid E_{1}\right)
$$

is surjective. Furthermore, by (2.9.3)

$$
\left(K_{S}+\phi^{*}\ulcorner M\urcorner-N\right)\left|E_{1} \equiv K_{E_{1}}+\left(\ulcorner R\urcorner E_{2}+\cdots+E_{k}\right)\right| E_{1} .
$$

Since the left-hand side of (2.11.1) is in any event an integer, we have in fact that ${ }^{\ulcorner} R^{\urcorner} \cdot E_{1} \geq 2$, and therefore

$$
\mathscr{O}_{S}\left(K_{S}+\phi^{*}\ulcorner M\urcorner-N\right) \mid E_{1}=\omega_{E_{1}} \otimes L,
$$

where $L$ is a line bundle of degree $\geq 2$ on the irreducible Gorenstein curve $E_{1}$. This implies (cf. [Hrt]) that $\mathscr{O}_{S}\left(K_{S}+\phi^{*}\ulcorner M\urcorner-N\right) \mid E_{1}$ is globally generated. But then as in (2.10) the theorem follows from the surjectivity $(* *)$. [When $x_{1}$ is smooth, the remarks at the end of $(2.10)$ cover the present situation as well if one bears in mind that $Z \cap E_{1}=\varnothing$ in the tricky case $\left[a_{0}+c r_{0}\right]=0$.] 
(2.12) Finally, we verify (2.11.1). By (2.9.2) and (2.9.1)

$$
\begin{aligned}
R \cdot E_{1} & =(1-c n) \phi^{*}(M) \cdot E_{1} \\
& \geq(1-c n) \beta_{1} \\
& >\left(1-\frac{1-\mu}{\beta_{2}} \sqrt{2}\right) \beta_{1},
\end{aligned}
$$

and the desired inequality follows from (2.3.1). Now suppose that $h^{-1}\left(x_{0}\right)=$ $\left\{x_{1}\right\}$. Then $E_{1}$ meets some component, say $Z^{\prime}$, of $Z$. Denoting by $a^{\prime}$ and $r^{\prime}$ the coefficients attached to $Z^{\prime}$ in (2.6), we have $\left[a^{\prime}+c r^{\prime}\right]=0$ since by the assumption of (2.11) $Z^{\prime}$ is not minimizing. Hence,

$$
{ }^{r} R^{\urcorner}=R+\left(a^{\prime}+c r^{\prime}\right) Z^{\prime}+\Delta^{\prime}
$$

for some effective divisor $\Delta^{\prime}$ which meets $E_{1}$ properly. Now

$$
a^{\prime} \geq \mu, \quad r^{\prime} \geq q_{1}>\frac{n \beta_{2}}{\sqrt{2}}, \quad \beta_{1} \geq 1-\mu \quad(\text { by }(2.3 .2)) .
$$

Hence,

$$
\begin{aligned}
r R^{\urcorner} \cdot E_{1} & >(1-c n) \beta_{1}+\mu+\frac{c n \beta_{2}}{\sqrt{2}} \\
& \geq(1-c n)(1-\mu)+\mu+\frac{c n \beta_{2}}{\sqrt{2}} \\
& \geq 1+c n\left(\mu-1+\frac{\beta_{2}}{\sqrt{2}}\right) .
\end{aligned}
$$

So as before the required inequality follows from the hypotheses (2.3.2) of the theorem. [When $x_{1}$ is smooth, the argument is similar.]

\section{MAIN THEOREM: STATEMENT AND SET-UP OF PROOF}

3.1. Notation and assumptions. In the following, $X$ is a smooth irreducible projective threefold, and

$$
h: X \longrightarrow X_{0}
$$

is a surjective birational morphism, with $X_{0}$ normal and projective. We consider a big and nef line bundle $B$ on $X$ such that

$$
B=B_{1}+h^{*} B_{0}
$$

for some nef line bundles $B_{0}$ and $B_{1}$ on $X_{0}$ and $X$ respectively. Assume that

$$
K_{X}+B=h^{*}\left(L_{0}\right) \quad \text { for some line bundle } L_{0} \text { on } X_{0} \text {. }
$$

Finally, fix a point $x_{0} \in X_{0}$ and rational numbers $\sigma_{3}>3$ and $\sigma_{2}, \sigma_{1}, \sigma_{0}>0$ such that:

$$
B^{3}>\left(\sigma_{3}\right)^{3}
$$

(3.1.4) $B^{2} \cdot S \geq\left(\sigma_{2}\right)^{2} \quad \forall$ surfaces $S \subset X$ s.t. $\operatorname{dim} h(S)=2$ and $x_{0} \in h(S)$;

$$
B \cdot C>\sigma_{1} \quad \forall \text { curves } C \subset X \text { s.t. } \operatorname{dim} h(C)=1 \text { and } x_{0} \in h(C) \text {; }
$$

(3.1.6) $\quad B_{0} \cdot C_{0}>\sigma_{0} \quad \forall$ curves $C_{0} \subset X_{0}$ s.t. $x_{0} \in C_{0}$. 
Our main result gives a numerical criterion for $L_{0}$ to have a nonvanishing section at $x_{0}$ :

3.2. Theorem. (I) In addition to $\sigma_{3}>3$, assume that the following inequalities hold:

$$
\begin{gathered}
\left(1-\frac{3}{\sigma_{3}}\right) \sigma_{2} \geq 2 ; \\
\left(1-\frac{2}{\sigma_{2}}-\frac{3}{\sigma_{3}}\right) \sigma_{1} \geq 1 ; \\
\left(1-\frac{3}{\sigma_{3}}\right) \sigma_{0} \geq 1 .
\end{gathered}
$$

Then $L_{0}$ is free at $x_{0}$. Equivalently, $\mathscr{O}_{X}\left(K_{X}+B\right)$ has a section which is nonvanishing at every point $x \in h^{-1}\left(x_{0}\right)$.

(II) Suppose furthermore that $h$ is an isomorphism over a neighborhood of $x_{0}$ or, in other words, that the fibre $h^{-1}\left(x_{0}\right)$ consists of a single point $x \in X$. Then the same conclusion holds with (3.2.1) and (3.2.2) replaced by

$$
\begin{aligned}
& \sigma_{2} \geq \max \left\{\frac{2 \sigma_{3}}{\sigma_{3}-1} \quad, \quad \frac{\sqrt{2} \sigma_{3}}{\sigma_{3}-2}\right\}, \\
& \sigma_{1} \geq \max \left\{\frac{2 \sigma_{3}}{\sigma_{3}-1} \quad, \quad \frac{\sigma_{3}}{\sigma_{3}-2}\right\},
\end{aligned}
$$

and with (3.2.3) unchanged.

3.3. Remark. If $\sigma_{3} \geq 3+\sqrt{2}$ then (3.2.4) and (3.2.5) are equivalent respectively to

$$
\sigma_{2} \geq \frac{2 \sigma_{3}}{\sigma_{3}-1}, \quad \sigma_{1} \geq \frac{2 \sigma_{3}}{\sigma_{3}-1} .
$$

We note also that the proof will show that one could take $B_{0}$ and $B_{1}$ to be $\mathbb{Q}$-Cartier $\mathbb{Q}$-divisors in (3.1.1), although of course $B$ must be Cartier.

3.4. Proof of results stated in Introduction. Theorem $1^{*}$ follows from 3.2(II) by taking $h=$ id and $B=B_{0}$; observe that we have combined (3.2.3) and (3.2.5) into a single inequality. If $B^{3} \geq 92$, then we can take $\sigma_{3}=\frac{9}{2}+\epsilon$ and Theorem 1 follows with some computation. In the situation of Proposition 4, Kawamata's work implies that there is a birational morphism $h: X \longrightarrow X_{0}$ to a projective normal threefold $X_{0}$ plus an ample line bundle $L_{0}$ on $X_{0}$, such that $\mathscr{O}_{X}\left(K_{X}+B\right)=h^{*} L_{0}$ (cf. [Fuj3, (0.4.15)]). If we take $B_{0}=6 L_{0}$ and $B_{1}=B$ then $B^{3} \geq B_{0}^{3}+B_{1}^{3} \geq 6^{3}+1$, so we can take $\sigma_{3}=6+\epsilon$ for small $\epsilon$. Then the inequalities in the theorem are satisfied with $\sigma_{i}=6-\epsilon_{i}(0 \leq i \leq 2)$ for suitable $\epsilon_{i}$. Similarly, when $K_{X}$ is big and nef, it pulls back from an ample line bundle $L_{0}$ on the canonical model $X_{0}$. Taking $B_{0}=6 L_{0}$ and $B_{1}=\mathscr{O}_{X}$, the inequalities for the first statement of Theorem 3 are checked using Matsuki's 
observation [Mat, Proposition 3(ii)] that $K_{X}^{3}$ is always even on a smooth threefold, so that we can take $\sigma_{3}=7<6 \sqrt[3]{2}$. The other statements are similar. The proof of Corollary $2^{*}$ occupies $\S 6$.

The remainder of this section is devoted to introducing the set-up for the proof of Theorem 3.2. The reader will note that we follow fairly closely some of the ideas in the proof of the Nonvanishing Theorem (cf. [KMM] or [CKM]). Beginning of Proof of Theorem 3.2.

(3.5) First, fix a point $x \in h^{-1}\left(x_{0}\right)$, and choose a small rational number $\sigma>0$ such that $B^{3}>\left(\sigma_{3} /(1-\sigma)\right)^{3}$. Since $B$ is big and nef, a well-known argument (cf. [SS, p. 146]) shows that $h^{0}(X, \mathscr{O}(n B))$ grows like $B^{3} n^{3} / 6$. Hence for $n \gg 0$ there exists a divisor

$$
D \in|n B| \quad \text { with } \quad q=\text { def }_{\text {mult }}(D)>\frac{n \sigma_{3}}{1-\sigma} .
$$

Next, as in [CKM, (8.7)], there is an effective divisor $D^{\prime} \subset X$ so that, for any sufficiently small $0<\epsilon \ll 1$, the $\mathbb{Q}$-divisor $B-\epsilon\left(D+D^{\prime}\right)$ is ample. Letting $D_{i}$ denote the irreducible components of $D+D^{\prime}$, we may therefore fix small positive rational numbers $d_{i}$ such that

$$
A^{\prime}=\frac{\sigma}{\sigma_{3}} B-\sum d_{i} D_{i} \quad\left(0<d_{i} \ll 1\right)
$$

is ample. We also write

$$
D=\sum s_{i} D_{i} \quad\left(s_{i} \geq 0\right) .
$$

Note that we are free to assume that the $d_{i}$ are arbitrarily small; in fact, we can replace each $d_{i}$ by $d_{i} / N$ for $N \gg 0$ while maintaining the required amplitude in (3.5.1).

(3.6) After first blowing up $x$, we perform a sequence of blowings-up along smooth centers to construct an embedded resolution

$$
f: Y \longrightarrow X
$$

of $D+D^{\prime}$. Thus supp $f^{*}\left(D+D^{\prime}\right)$ is supported on a normal crossing divisor $\sum E_{j}$. Put

$$
f^{*} D=\sum r_{j} E_{j}, \quad K_{Y}-f^{*} K_{X}=\sum b_{j} E_{j} .
$$

Denote by

$$
g=h \circ f: Y \longrightarrow X_{0}
$$

the composition of $f$ with the given map $h$.

(3.7) For each of the divisors $E_{j}$ which is $f$-exceptional fix a small positive rational number $e_{j}$ in such a way that the $\mathbb{Q}$-line bundle

$$
A=f^{*}\left(A^{\prime}\right)-\sum_{\substack{E_{j} \\ f \text {-exceptional }}} e_{j} E_{j} \quad\left(0<e_{j} \ll 1\right)
$$


is ample. We then define rational numbers $\delta_{j}>0$ via

$$
f^{*}\left(\sum d_{i} D_{i}\right)+\sum^{\prime} e_{j} E_{j}=\sum \delta_{j} E_{j},
$$

where the second sum on the left is taken over those $E_{j}$ which are $f$-exceptional. ${ }^{1}$ Thus $A=f^{*}\left(\sigma B / \sigma_{3}\right)-\sum \delta_{j} E_{j}$.

(3.8) Now set

$$
c_{j}=\frac{b_{j}+1-\delta_{j}}{r_{j}} .
$$

(We put $c_{j}=\infty$ if $r_{j}=0$.) Varying the $d_{i}$ and $e_{j}$ slightly if necessary, while preserving (3.7.2), we may suppose that all the (finite) $c_{j}$ are distinct. Let

$$
c=\min \left\{c_{j} \mid E_{j} \cap g^{-1}\left(x_{0}\right) \neq \varnothing, r_{j}>0\right\} .
$$

We assume that the minimum occurs for $j=0$, and we write

$$
E=E_{0}, \quad r=r_{0}, \quad b=b_{0}, \quad \delta=\delta_{0} .
$$

Thus $g(E) \ni x_{0}$, and $b-c r-\delta=-1$. We call $E$ the minimizing component, and we adopt the convention that unless otherwise mentioned sums of the form $\sum n_{j} E_{j}$ are assumed taken over $j \geq 1$. Observe that we have arranged things so that $b_{j}-c r_{j}-\delta_{j}>-1$ for all $j \neq 0$ such that $E_{j}$ meets $g^{-1}\left(x_{0}\right)$.

By examining the coefficients attached to the exceptional divisor created by blowing up $x$, we see that $c n<3 / \sigma_{3}-3 \sigma / \sigma_{3}$. Thus

$$
\left(1-c n-\frac{\sigma}{\sigma_{3}}\right)>\left(1-\frac{3}{\sigma_{3}}+\frac{2 \sigma}{\sigma_{3}}\right)>0,
$$

and in particular $\left(1-c n-\sigma / \sigma_{3}\right) f^{*} B+A$ is ample.

(3.9) Consider now the $\mathbb{Q}$-divisor on $Y$ :

$$
R=\sum_{j \geq 1}\left(b_{j}-c r_{j}-\delta_{j}\right) E_{j}
$$

We can write

$$
{ }^{r} R^{\top}=P-N,
$$

where $P$ and $N$ are effective divisors without common components, meeting $E$ properly. Note that all the components of $P$ are $f$-exceptional (since $b_{j}>0$ for such a component), and by construction

$$
\operatorname{supp}(N) \cap g^{-1}\left(x_{0}\right)=\varnothing .
$$

Put

$$
M=f^{*}\left(K_{X}+B\right)-K_{Y}-E+R .
$$

\footnotetext{
${ }^{1}$ A word to the experts: as usual, the $\delta_{j}$ will play the role of "perturbation variables" to ensure a unique minimizing component in (3.8). However, it will be important in $\S 5$ to have some control over which a component gets picked in case of a tie. This is why we go through the present construction.
} 
Then

$$
M \equiv f^{*}\left(\left(1-c n-\frac{\sigma}{\sigma_{3}}\right) B\right)+A,
$$

and hence $M$ is ample. The Kawamata-Viehweg vanishing theorem therefore yields

Setting

$$
H^{1}\left(Y, \mathscr{O}_{Y}\left(f^{*}\left(K_{X}+B\right)-E+P-N\right)\right)=0 .
$$

$$
\bar{f}=f|E, \quad \bar{g}=g| E, \quad N^{\prime}=N\left|E, \quad P^{\prime}=P\right| E,
$$

this means that the restriction map

$$
H^{0}\left(Y, \mathscr{O}_{Y}\left(f^{*}\left(K_{X}+B\right)+P-N\right)\right) \longrightarrow H^{0}\left(E, \mathscr{O}_{E}\left(\bar{f}^{*}\left(K_{X}+B\right)+P^{\prime}-N^{\prime}\right)\right)
$$

is surjective.

(3.10) It will be convenient to remove the positive components $P$ from (3.9.7). To this end, recall first the standard

3.10.1. Lemma. Suppose that $h: V \rightarrow W$ is a proper birational morphism of smooth projective varieties. Let $Q$ and $R$ be effective divisors on $V$ having no common components, and assume that every component of $Q$ is h-exceptional. Then $h_{*}\left(\mathscr{O}_{Q}(Q)\right)=h_{*}\left(\mathscr{O}_{Q}(Q-R)\right)=0$, and consequently $h_{*}\left(h^{*}(\mathscr{E})(Q-R)\right)=$ $h_{*}\left(h^{*}(\mathscr{E})(-R)\right)$ for any locally free sheaf $\mathscr{E}$ on $W$.

3.11. Lemma. In the commutative diagram

$$
\begin{array}{ccc}
H^{0}\left(Y, \mathscr{O}_{Y}\left(f^{*}\left(K_{X}+B\right)-N\right)\right) & \rightarrow & H^{0}\left(E, \mathscr{O}_{E}\left(\bar{f}^{*}\left(K_{X}+B\right)-N^{\prime}\right)\right) \\
\cdot P \downarrow & \downarrow \cdot P^{\prime} \\
H^{0}\left(Y, \mathscr{O}_{Y}\left(f^{*}\left(K_{X}+B\right)+P-N\right)\right) & \rightarrow H^{0}\left(E, \mathscr{O}_{E}\left(\bar{f}^{*}\left(K_{X}+B\right)+P^{\prime}-N^{\prime}\right)\right),
\end{array}
$$

both vertical maps are isomorphisms and both horizontal maps are surjective.

Proof. The bottom horizontal map is surjective by (3.9.7), and the vertical map on the left is an isomorphism thanks to Lemma 3.10.1. Since the vertical map on the right is in any event injective, the assertion follows.

(3.12) In conclusion, we observe that in order to prove the theorem, it is now enough to verify the following

3.12.1. Criterion. There is a point $y \in \bar{g}^{-1}\left(x_{0}\right)$ and a section

$$
\bar{s} \in H^{0}\left(E, \bar{f}^{*}\left(K_{X}+B\right)\left(-N^{\prime}\right)\right),
$$

such that $\bar{s}(y) \neq 0$.

Proof that (3.12.1) $\Rightarrow$ (3.2). By (3.11), $\bar{s}$ lifts to some

$$
s \in H^{0}\left(Y, f^{*} \mathscr{O}_{X}\left(K_{X}+B\right)(-N)\right)
$$

not vanishing at $y$. Since $N \cap g^{-1}\left(-x_{0}\right)=\varnothing, s$ in turn determines a section

$$
t \in H^{0}\left(Y, f^{*} \mathscr{O}_{X}\left(K_{X}+B\right)\right) \text { with } t(y) \neq 0 .
$$


But since $g$ is birational and $X_{0}$ is normal,

$$
H^{0}\left(Y, f^{*} \mathscr{O}_{X}\left(K_{X}+B\right)\right)=H^{0}\left(X_{0}, L_{0}\right) .
$$

So (3.12.1) indeed implies the existence of the desired section.

The plan now is to proceed in cases. In $\S 4$ we verify $(3.12 .1)$ when dim $g(E) \leq 1$. Section 5 is devoted to the case $\operatorname{dim} g(E)=2$.

\section{The CASE OF AN EXCEPTIONAL MINIMIZING COMPONENT}

Keeping notation as in $\S 3$, we complete the proof when the minimizing component $E \subset Y$ maps via $g: Y \longrightarrow X_{0}$ to a variety of dimension $\leq 1$.

(4.1) Suppose first that $\operatorname{dim} g(E)=0$. Then $g(E)=x_{0}$, and so $N^{\prime}=N \mid E=$ $\mathscr{O}_{E}$ thanks to $(3.9 .3)$. Hence

$$
\bar{f}^{*} \mathscr{O}_{X}\left(K_{X}+B\right)\left(-N^{\prime}\right)=\bar{g}^{*}\left(L_{0}\right)=\mathscr{O}_{E} .
$$

So Criterion (3.12.1) is clear in this case.

(4.2) Assume henceforth that $C_{0}=_{\text {def }} g(E) \subset X_{0}$ is a curve passing through $x_{0}$. Let

$$
E \stackrel{\bar{\rho}}{\longrightarrow} C \stackrel{\bar{\nu}}{\longrightarrow} X_{0}
$$

be the Stein factorization of $\bar{g}$. Observe that $C$ is normal and hence smooth and that $\bar{\rho}$ is flat. Fix a point $Q \in C$ over $x_{0}$, and let $Z=\bar{\rho}^{-1}(Q)$, so that $Z \subset E$ is a divisor, the scheme-theoretic fibre of $\bar{\rho}$ over $Q$.

(4.3) With notation as in $\S 3$, the strategy now will be to use Kawamata-Viehweg vanishing on $E$ to prove the following

Assertion 4.3.1. There is a section $\bar{s} \in \Gamma\left(E, \mathscr{O}_{E}\left(\bar{f}^{*}\left(K_{X}+B\right)-N^{\prime}\right)\right)$ whose image under the restriction map

$$
H^{0}\left(E, \mathscr{O}_{E}\left(\bar{f}^{*}\left(K_{X}+B\right)-N^{\prime}\right)\right) \longrightarrow H^{0}\left(Z, \mathscr{O}_{E}\left(\bar{f}^{*}\left(K_{X}+B\right)-N^{\prime}\right) \mid Z\right)
$$

is nonzero.

(4.4) We check that (4.3) implies Criterion (3.12.1). In fact, recall from (3.1.2) that $\bar{f}^{*} \mathscr{O}_{X}\left(K_{X}+B\right)=\bar{g}^{*}\left(L_{0}\right)$, and note that $\bar{\rho}_{*} \mathscr{O}_{E}=\mathscr{O}_{C}$. Therefore, one has an isomorphism:

$$
H^{0}\left(C, \bar{\nu}^{*} L_{0}\right) \stackrel{\bar{\rho}^{*}}{\simeq} H^{0}\left(E, \mathscr{O}_{E}\left(\bar{f}^{*}\left(K_{X}+B\right)\right)\right) .
$$

Thus, viewing $\bar{s}$ as a section of $\mathscr{O}_{E}\left(\bar{f}^{*}\left(K_{X}+B\right)\right)$ vanishing on $N^{\prime}$, we have $\bar{s}=\bar{\rho}^{*}(\bar{t})$ for some $\bar{t} \in H^{0}\left(C, \bar{\nu}^{*} L_{0}\right)$. Then $\bar{t}(Q) \neq 0$ or else $\bar{s}$ would vanish on the scheme $Z$. Therefore, $\bar{s}$ is nonvanishing on $\operatorname{supp} Z$, and (3.12.1) is verified.

(4.5) Turning to the proof of (4.3.1), the first point is to establish the vanishing:

$$
H^{1}\left(E, \mathscr{O}_{E}\left(\bar{f}^{*}\left(K_{X}+B\right)+P^{\prime}-N^{\prime}-Z\right)\right)=0 .
$$


In fact, referring to (3.9), set $R^{\prime}=R \mid E$, so that $\left.{ }^{r} R^{\prime}\right\urcorner=P^{\prime}-N^{\prime}$, and let $M^{\prime}=$ $\bar{f}^{*}\left(K_{X}+B\right)-K_{E}+R^{\prime}=M \mid E$. Consider on $E$ the $\mathbb{Q}$-divisor $L=M^{\prime}-Z$. The sheaf appearing in (4.5.1) is $\left.\mathscr{O}_{E}\left(K_{E}+{ }^{r} L\right\urcorner\right)$, and hence (4.5.1) will follow if we prove that $L$ is ample. To this end, set $\gamma=\left(1-c n-\sigma / \sigma_{3}\right)$. Then

$$
\begin{aligned}
L & \equiv \bar{f}^{*}(\gamma B)+(A \mid E)+\bar{\rho}^{*} \mathscr{O}_{C}(-Q) \quad(\text { by }(3.9 .5)) \\
& \equiv \bar{g}^{*}\left(\gamma B_{0}\right)+\bar{\rho}^{*} \mathscr{O}_{C}(-Q)+\left(\bar{f}^{*}\left(\gamma B_{1}\right)+A \mid E\right) \quad(\text { by }(3.1 .1)) \\
& \equiv \bar{\rho}^{*}\left(\bar{\nu}^{*}\left(\gamma B_{0}\right) \otimes \mathscr{O}_{C}(-Q)\right)+\left(\bar{f}^{*}\left(\gamma B_{1}\right)+A \mid E\right) .
\end{aligned}
$$

Now by (3.1.6)

$$
\operatorname{deg}_{C}\left(\bar{\nu}^{*}\left(\gamma B_{0}\right) \otimes \mathscr{O}_{C}(-Q)\right) \geq \gamma \cdot \sigma_{0}-1 .
$$

But $\gamma \geq\left(1-3 / \sigma_{3}\right)$ by $(3.8 .1)$, and $\left(1-3 / \sigma_{3}\right) \sigma_{0} \geq 1$ by (3.2.3). Thus

$$
\bar{\rho}^{*}\left(\bar{\nu}^{*}\left(\gamma B_{0}\right) \otimes \mathscr{O}_{C}(-Q)\right)
$$

is nef. On the other hand, $\bar{f}^{*}\left(\gamma B_{1}\right)+A \mid E$ is ample since $B_{1}$ is nef and $A$ is ample. Hence $L$ too is ample, and (4.5.1) is proved.

(4.6) We claim next that

$$
H^{0}\left(Z, \mathscr{O}_{E}\left(\bar{f}^{*}\left(K_{X}+B\right)+P^{\prime}-N^{\prime}\right) \mid Z\right) \neq 0 .
$$

In fact, consider on $E$ the line bundle $M_{1}=\mathscr{O}_{E}\left(\bar{f}^{*}\left(K_{X}+B\right)+P^{\prime}-N^{\prime}\right)$. By (3.9.3), $N^{\prime} \cap Z=\varnothing$, and consequently no component of $N^{\prime}$ dominates $C$ under $\bar{\rho}: E \longrightarrow C$. Therefore, if $Z^{\prime}=\bar{\rho}^{-1}\left(Q^{\prime}\right)$ is the fibre of $\bar{\rho}$ over a general point $Q^{\prime} \in C$, we have $M_{1} \mid Z^{\prime} \cong \mathscr{O}_{Z^{\prime}}\left(P^{\prime} \mid Z^{\prime}\right)$. In particular, $H^{0}\left(Z^{\prime}, M_{1} \mid Z^{\prime}\right) \neq 0$ and (4.6.1) follows by semicontinuity.

(4.7) Consider finally the commutative diagram

$$
\begin{gathered}
H^{0}\left(E, \mathscr{O}_{E}\left(\bar{f}^{*}\left(K_{X}+B\right)\right)\left(-N^{\prime}\right)\right) \rightarrow H^{0}\left(Z, \mathscr{O}_{E}\left(\bar{f}^{*}\left(K_{X}+B\right)\right)\left(-N^{\prime}\right) \mid Z\right) \\
\cdot P^{\prime} \downarrow \\
\quad \downarrow \cdot P^{\prime} \\
H^{0}\left(E, \mathscr{O}_{E}\left(\bar{f}^{*}\left(K_{X}+B\right)\right)\left(P^{\prime}-N^{\prime}\right)\right) \rightarrow H^{0}\left(Z, \mathscr{O}_{E}\left(\bar{f}^{*}\left(K_{X}+B\right)\right)\left(P^{\prime}-N^{\prime}\right) \mid Z\right)
\end{gathered}
$$

The left vertical map is an isomorphism by (3.11), and the lower horizontal map is surjective thanks to (4.5.1). Assertion (4.3.1) then follows from (4.6.1).

This completes the proof of the theorem in the cases $\operatorname{dim} g(E) \leq 1$.

(4.8) Remark. Note that among the numerical hypotheses of Theorem 3.2, we have so far only used the inequality (3.2.3).

\section{NONEXCEPTIONAL MINIMIZING COMPONENTS}

This section is devoted to the remaining case, when the minimizing component $E$ maps to a surface $g(E) \subset X_{0}$. In principle one would like to apply the $\mathbb{Q}$-Reider Theorem 2.3 on $S=f(E) \subset X$ to produce the section required in Criterion (3.12.1). However, there are various technical complications here; e.g., $S$ might be very singular, and one would have to relate the divisor $N$ on $E$ to something on $S$. Happily we are able to control the geometry of $S$ along $g^{-1}\left(x_{0}\right)$, and this lets us construct an intermediate surface where (2.3) indeed applies. 
(5.1) We start with some notation supplementing (3.9). To begin with, decompose the divisor $P$ into a sum $P=P_{1}+P_{2}$, where $P_{1}$ consists of those components of $P$ that meet $g^{-1}\left(x_{0}\right)$ and $P_{2}$ is formed from the others. Thus $g\left(E^{\prime}\right) \ni x_{0}$ for every component $E^{\prime}$ of $P_{1}$ and

$$
\operatorname{supp}\left(P_{2}\right) \cap g^{-1}\left(x_{0}\right)=\varnothing .
$$

Next, if $T$ is a $\mathbb{Q}$-divisor on $Y$ not containing $E$, denote by $T^{\prime}$ the restriction of $T$ to $E$. Thus

$$
R^{\prime}=R\left|E, \quad P_{i}^{\prime}=P_{i}\right| E, \quad N^{\prime}=N\left|E, \quad E_{j}^{\prime}=E_{j}\right| E \quad(\text { for } j \geq 1) .
$$

Similarly, we set $M^{\prime}=\bar{f}^{*}\left(K_{X}+B\right)-K_{E}+R^{\prime}$.

(5.2) Keeping the notation of $\S 3$, we assume henceforth that $S_{0}={ }_{\text {def }} g(E) \subset$ $X_{0}$ is a surface through $x_{0}$. In this case, $E$ is the proper transform in $Y$ of a surface $S \subset X$ appearing as one of the components of $D$ in (3.5.2), say $S=D_{0}$. Thus,

$$
r=\operatorname{ord}_{E}\left(f^{*} D\right)=s_{0}>0, \quad \delta=d_{0}, \quad \text { and } \quad c=\frac{1-d_{0}}{r} .
$$

Let $\bar{h}: S \longrightarrow S_{0}$ be the natural map, and set $Z=\bar{h}^{-1}\left(x_{0}\right)$. The situation is summarized in the following diagram:

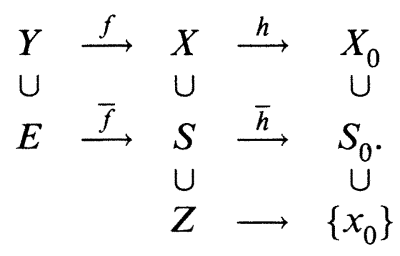

Recall that $g=h \circ f$ and $\bar{g}=\bar{h} \circ \bar{f}$.

(5.3) We need to say something about the geometry of $S$. To this end, define integers $t_{j} \geq 0$ by writing

$$
f^{*}(S)=E+\sum_{j \geq 1} t_{j} \cdot E_{j}
$$

5.3.2. Lemma. Fix one of the divisors $E_{j} \subset Y$ other than $E$ itself.

(i) If $\operatorname{ord}_{E_{j}}(P)>0$ then $b_{j}>t_{j}$.

(ii) If $E_{j} \cap g^{-1}\left(x_{0}\right) \neq \varnothing$ then $b_{j} \geq t_{j}$.

Proof. If $t_{j}=0$, both assertions are clear, so assume $t_{j}>0$. Then $E_{j}$ is $f$-exceptional; hence taking $i=0$ in (3.7.2) gives

$$
\delta_{j} \geq e_{j}+d_{0} \cdot t_{j}>d_{0} \cdot t_{j} .
$$

On the other hand, it follows in any event from (5.3.1) and (3.5.2) that

$$
r_{j}=\operatorname{ord}_{E_{j}}\left(f^{*} D\right) \geq r \cdot t_{j} \text {. }
$$


Now $\operatorname{ord}_{E_{j}}(P)>0$ if and only if $b_{j}-c r_{j}-\delta_{j}>0$. In this case

$$
\begin{aligned}
b_{j}>\delta_{j}+c r_{j} & =\delta_{j}+\left(\frac{1-d_{0}}{r}\right) r_{j} \\
& \geq \delta_{j}+\left(1-d_{0}\right) t_{j} \quad \text { by }(5.3 .4) \\
& >t_{j} \quad \text { by }(5.3 .3) .
\end{aligned}
$$

For (ii) suppose that $x_{0} \in g\left(E_{j}\right)$. Then $c_{j}=\left(b_{j}+1-\delta_{j}\right) / r_{j}>\left(1-d_{0}\right) / r=c$, which as above yields $b_{j}+1>t_{j}$. The assertion follows.

Now we come to an essential point (compare [W2] and [Kol3, 17.6]):

5.4. Proposition. Fix any point $z \in Z$. Then:

(i) $S$ is normal in a neighborhood of $z$.

(ii) $S$ is either smooth at $z$ or has a rational double point at $z$.

(iii) Every component of $P_{1}^{\prime}$ is $\bar{f}$-exceptional.

Proof. For (i) it is equivalent to show that $S$ is smooth in codimension one in a neighborhood of $z$. Suppose to the contrary that $\operatorname{Sing}(S) \supset \Gamma \ni z$ is a curve of singularities. Then at some stage in the construction of the embedded resolution, we would have to blow up (a proper transform of) $\Gamma$. The first such blowing-up gives rise to an exceptional divisor $E^{\prime} \subset Y$ with $x_{0} \in g\left(E^{\prime}\right)$; denote by $b^{\prime}$ and $t^{\prime}$ the corresponding coefficients. Then $b^{\prime}=1$ and $t^{\prime}=\operatorname{mult}_{\Gamma}(S) \geq$ 2. But this contradicts 5.3.2(ii).

For (ii) and (iii), note first that

$$
K_{Y}+E \equiv f^{*}\left(K_{X}+S\right)+\sum_{j \geq 1}\left(b_{j}-t_{j}\right) E_{j}
$$

Therefore,

$$
K_{E} \equiv \bar{f}^{*}\left(K_{S}\right)+\sum_{j \geq 1}\left(b_{j}-t_{j}\right) E_{j}^{\prime}
$$

Moreover, an elementary residue calculation (cf. [Kmf, p. 180]) shows that at least over the normal locus of $S,(5.4 .1)$ computes $K_{E / S}$ on the level of divisors. (In other words, given a normal point $w \in S$ and $y \in \bar{f}^{-1}(w)$ we can find local generators $\eta \in \omega_{S, w}$ and $\eta^{\prime} \in \omega_{E, y}$ such that $f^{*}(\eta)=\phi \cdot \eta^{\prime}$, where $\left.\operatorname{div}(\phi)={ }_{\text {near } y} \sum\left(b_{j}-t_{j}\right) E_{j}^{\prime}.\right)$ In particular, if $\bar{f}\left(E_{j}^{\prime}\right) \ni z$ and $b_{j}>t_{j}$, then $E_{j}^{\prime}$ must be $\bar{f}$-exceptional. Statement (iii) then follows at once from 5.3.2(i). Similarly, (5.4.1) and 5.3.2(ii) imply that $S$ has at worst a canonical singularity at $z$, whence (ii).

(5.5) We next factor $\bar{f}$ through a "partial resolution" of $S$. Specifically, we construct a surface $S_{1}$ sitting in the diagram:

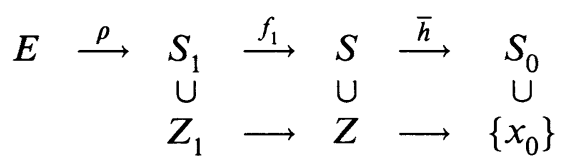


with $\bar{f}=f_{1} \circ \rho$ and $Z_{1}=f_{1}^{-1}(Z)$, such that:

$f_{1}$ is an isomorphism over a neighborhood of $Z$;

$$
\rho \text { is an isomorphism over } S_{1}-T_{1} \text { for a finite set } T_{1} \subset Z_{1} \text {. }
$$

For the existence of such a factorization, note that since $S$ is normal along $Z$, there is a neighborhood $V$ of $Z$ in $S$ such that $\bar{f}^{-1}(V) \rightarrow V$ is an isomorphism over the complement of a finite set $T \subset Z$. We may then contract $\bar{f}^{-1}(T) \subset E$ to obtain $S_{1}$. Set

$$
h_{1}=\bar{h} \circ f_{1}: S_{1} \longrightarrow S_{0} \text {. }
$$

By abuse of notation, we will also view $f_{1}$ as a map $f_{1}: S_{1} \longrightarrow X$. Observe that $S_{1}$ is smooth except perhaps for finitely many rational double points along $Z_{1}$. In particular, $S_{1}$ is Gorenstein and $\mathbb{Q}$-factorial.

(5.6) Consider now the $\mathbb{Q}$-divisor $R_{1}=\rho_{*}\left(R^{\prime}\right)$ on $S_{1}$, where $\rho_{*}: \operatorname{Div}_{\mathbb{Q}}(E)$ $\rightarrow \operatorname{Div}_{\mathbb{Q}}\left(S_{1}\right)$ is the divisor-theoretic push-forward determined by $\rho$, and put

$$
M_{1}=f_{1}^{*}\left(K_{X}+B\right)-K_{S_{1}}+R_{1} \text {. }
$$

The plan is to apply the Reider-type Theorem 2.3 to $M_{1}$ and to the map $h_{1}$ : $S_{1} \longrightarrow S_{0}$.

(5.7) The first point is to analyze the divisor $\left.K_{S_{1}}+{ }^{r} M_{1}\right\urcorner$. To this end, observe that since $\rho$ is obtained by contracting finitely many curves in $E, \rho_{*}$ commutes with rounding. Therefore, $\left.{ }^{\ulcorner} R_{1}\right\urcorner=\rho_{*}\left(\left\ulcorner R^{\prime}\right\urcorner\right)=\rho_{*}\left(P^{\prime}\right)-\rho_{*}\left(N^{\prime}\right)$. Now $\rho_{*}\left(P_{1}^{\prime}\right)=0$ since $P_{1}^{\prime}$ is $\bar{f}$-exceptional [5.4(iii)]. So writing

$$
P_{2}^{\prime \prime}=\rho_{*}\left(P_{2}^{\prime}\right), \quad N^{\prime \prime}=\rho_{*}\left(N^{\prime}\right),
$$

we have

$$
\left.K_{S_{1}}+{ }^{r} M_{1}\right\urcorner=f_{1}^{*}\left(K_{X}+B\right)+P_{2}^{\prime \prime}-N^{\prime \prime} .
$$

But the divisors $P_{2}^{\prime}$ and $N^{\prime}$ are supported on the complement of $\bar{g}^{-1}\left(x_{0}\right)$ by (5.1.1) and (3.9.2), and hence

$$
\operatorname{Supp}\left(P_{2}^{\prime \prime}\right) \cap Z_{1}=\varnothing, \quad \operatorname{Supp}\left(N^{\prime \prime}\right) \cap Z_{1}=\varnothing .
$$

It follows in the first place that $P_{2}^{\prime \prime}$ and $N^{\prime \prime}$ are supported in the smooth locus of $S_{1}$, and hence ${ }^{r} M_{1}{ }^{\top}$ is Cartier. Suppose now that $\Gamma \subset S_{1}$ is a reduced curve (possibly reducible) such that $h_{1}(\Gamma)=x_{0}$. Then by (5.7.1), (5.7.2), and (3.1.2)

$$
\left.\mathscr{O}_{S_{1}}\left(K_{S_{1}}+{ }^{r} M_{1}\right\urcorner\right)\left|\Gamma \cong \mathscr{O}_{S_{1}}\left(f_{1}^{*}\left(K_{X}+B\right)\right)\right| \Gamma \cong h_{1}^{*}\left(L_{0}\right) \mid \Gamma \cong \mathscr{O}_{\Gamma} .
$$

Hence the first hypothesis of Theorem 2.3 is satisfied.

(5.8) We verify next that $M_{1}$ satisfies the numerical hypotheses (2.3.1) with $\mu=0$. Since $\rho_{*}$ is compatible with rational equivalence and $\rho_{*}\left(K_{E}\right)=K_{S_{1}}$, we find

$$
M_{1} \equiv \rho_{*}\left(\bar{f}^{*}\left(K_{X}+B\right)-K_{E}+R^{\prime}\right) \equiv \rho_{*}\left(M^{\prime}\right)
$$


Set $\gamma=\left(1-c n-\sigma / \sigma_{3}\right)$. Then $M^{\prime} \equiv \bar{f}^{*}(\gamma B)+A \mid E$; whence,

$$
M_{1} \equiv f_{1}^{*}(\gamma B)+\rho_{*}(A \mid E) \text {. }
$$

Now $\rho_{*}(A \mid E)$, being the push-forward of a nef divisor under a proper surjective morphism of $\mathbb{Q}$-factorial surfaces, is nef. Hence $M_{1}$ is nef. Furthermore, by Kleiman's theorem, two nef divisors have a nonnegative intersection number. Therefore,

$$
M_{1} \cdot M_{1} \geq \gamma^{2}\left(B^{2} \cdot S\right) \underset{(3.1 .4)}{>} \gamma^{2}\left(\sigma_{2}\right)^{2}
$$

Similarly, for any curve $\Gamma \subset S_{1}$ such that $h_{1}(\Gamma)$ is a curve through $x_{0}$

$$
M_{1} \cdot \Gamma \geq \gamma\left(\left(f_{1}\right)_{*}[\Gamma] \cdot B\right) \underset{(3.1 .5)}{>} \gamma \cdot \sigma_{1} \text {. }
$$

Thus we can take

$$
\beta_{2}=\left(1-c n-\frac{\sigma}{\sigma_{3}}\right) \sigma_{2} \text { and } \beta_{1}=\left(1-c n-\frac{\sigma}{\sigma_{3}}\right) \sigma_{1}
$$

in (2.2). Since $\gamma>\left(1-3 / \sigma_{3}\right)$ [by (3.8.1)], it is enough for (2.3.1) to show

$$
\left(1-\frac{3}{\sigma_{3}}\right) \sigma_{2} \geq 2 \text { and } \sigma_{1}\left(1-\frac{3}{\sigma_{3}}\right)\left(1-\frac{2}{\left(1-\frac{3}{\sigma_{3}}\right) \sigma_{2}}\right) \geq 1 \text {. }
$$

But this is exactly the content of the inequalities (3.2.1) and (3.2.2) in the hypotheses of statement (I) of the theorem.

(5.9) We conclude from Theorem 2.3 and (5.7.1) the existence of a section

$$
s_{1} \in H^{0}\left(S_{1}, \mathscr{O}_{S_{1}}\left(f_{1}^{*}\left(K_{X}+B\right)+P_{2}^{\prime \prime}-N^{\prime \prime}\right)\right)
$$

which is nonvanishing at some point $x_{1} \in Z_{1}$. But $\rho$ is an isomorphism over the supports of $P_{2}^{\prime \prime}$ and $N^{\prime \prime}$ [by (5.5.3)], and consequently $\rho^{*}\left(P_{2}^{\prime \prime}\right)=P_{2}^{\prime}$, $\rho^{*}\left(N^{\prime \prime}\right)=N^{\prime}$. Thus

$$
\bar{s}_{1}=_{\text {def }} \rho^{*}\left(s_{1}\right) \in H^{0}\left(E, \mathscr{O}_{E}\left(\bar{f}^{*}\left(K_{X}+B\right)+P_{2}^{\prime}-N^{\prime}\right)\right)
$$

is a section of the indicated bundle which is nonvanishing at some point $y \in$ $\bar{g}^{-1}\left(x_{0}\right)$. Consider then the commutative diagram

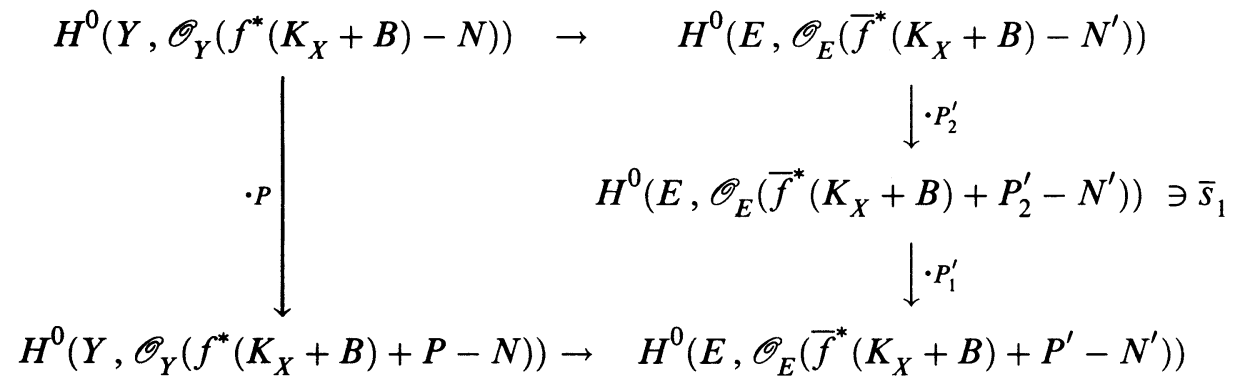

By (3.11) the vertical homomorphism on the left is an isomorphism and the horizontal maps are surjective. Since the vertical maps on the right are in any event injective, it follows from the diagram that they are bijective. The section 
$\bar{s} \in H^{0}\left(E, \mathscr{O}_{E}\left(\bar{f}^{*}\left(K_{X}+B\right)-N^{\prime}\right)\right)$ then mapping to $\bar{s}_{1}$ is nonvanishing at $y$, and therefore Criterion (3.12.1) is verified.

This completes the proof of statement (I) of Theorem 3.2.

5.10. Remark. The argument just given leads to a variant in the situation of statement (II) of the theorem. Specifically, suppose that $h$ is an isomorphism over $x_{0}$. Assume that the numerical hypotheses (3.2.1) and (3.2.3) hold, but that $(3.2 .2)$ is replaced by

$$
\left(1-\frac{3}{\sigma_{3}}\right) \sigma_{1} \geq 2
$$

Then $L_{0}$ is free at $x_{0}$. In fact, just as in (5.8), one can use (5.10.1) and (3.2.2) to verify the numerical hypotheses (2.3.2) of Theorem 2.3 with $\mu=0$. In the situation of Fujita's conjecture, for example, this leads to the statement that $K_{X}+6 A$ is base-point free if $A$ is ample. However, in the following paragraphs we will see that with more work one can do a little better by using a nontrivial estimate for $\mu$.

(5.11) Turning to statement (II), assume now that $h^{-1}\left(x_{0}\right)$ consists of the single point $x \in X$ appearing in (3.5). Then $S$ passes through $x$, and we denote by $x_{1} \in S_{1}$ the point lying over $x$. Put

$$
\left.\Delta_{1}={ }^{\ulcorner} M_{1}\right\urcorner-M_{1}=\rho_{*}\left(\left\ulcorner R^{\prime}\right\urcorner-R^{\prime}\right),
$$

and let $\mu=$ mult $_{x_{1}}\left(\Delta_{1}\right)$. It is enough to show that the numerical hypotheses (2.3.2) of Theorem 2.3 are satisfied with $\beta_{1}$ and $\beta_{2}$ as given in (5.8.1).

To this end, we need to estimate $\mu$. Let $D^{*}=\sum_{i \geq 1} s_{i} \cdot D_{i}$ be the part of $D$ excluding $D_{0}=S$, so that $D=r S+D^{*}$. The essential point is

5.12. Lemma. As in (3.5), let $q=$ mult $_{x}(D)$.

(i) There is a neighborhood $V_{1}$ of $x_{1}$ in $S_{1}$ such that $f_{1}^{*}\left(c D^{*}\right) \mid V_{1} \leq$ $\Delta_{1} \mid V_{1}$.

(ii) If $x_{1}$ is a smooth point of $S_{1}$, then $\mu \geq\left(1-d_{0}\right)(q-r) / r$.

(iii) If $x_{1}$ is an $R D P$ of $S_{1}$, then $\mu \geq\left(1-d_{0}\right)(q-2 r) / r$.

Proof. Since mult ${ }_{x}\left(D^{*}\right)=q-r \cdot$ mult $_{x}(S)$, statements (ii) and (iii) follow from (i) by virtue of (2.1.1). For (i) note to begin with that:

$$
f^{*}\left(c D^{*}\right)=f^{*}(c D-c r S)=\sum_{j \geq 1}\left(c r_{j}-\left(1-d_{0}\right) t_{j}\right) E_{j}
$$

Next, choose a small neighborhood $V$ of $x$ in $S$ such that the following hold:

(a) $f_{1}$ is an isomorphism over $V$ (so $S$ is smooth on $V$ except perhaps at $x$ ).

(b) If $V_{1}=f_{1}^{-1}(V)$ and $V_{2}=\rho^{-1}\left(V_{1}\right)$, then $\operatorname{supp}\left(P_{2}^{\prime}\right) \cap V_{2}=\varnothing$ and $\operatorname{supp}\left(N^{\prime}\right) \cap V_{2}=\varnothing$.

By (3.9.1) and (3.9.2)

$$
\left.{ }^{\ulcorner} R^{\prime}\right\urcorner-R^{\prime}=P^{\prime}-N^{\prime}+\sum\left(\delta_{j}+c r_{j}-b_{j}\right) E_{j}^{\prime} .
$$


But $\left(P^{\prime}-N^{\prime}\right)\left|V_{2}=P_{1}^{\prime}\right| V_{2}$ thanks to (b), and writing

$$
\left(\delta_{j}+c r_{j}-b_{j}\right)=\left(c r_{j}-\left(1-d_{0}\right) t_{j}\right)+\left(t_{j}-b_{j}\right)+\left(\delta_{j}-d_{0} t_{j}\right)
$$

we find from $(*)$ and $(5.4 .1)$

$$
\left(\left\ulcorner R^{\prime}\right\urcorner-R^{\prime}\right)\left|V_{2}=\left(P_{1}^{\prime}+\bar{f}^{*}\left(c D^{*}\right)-K_{E / S}+\sum\left(\delta_{j}-d_{0} t_{j}\right) E_{j}^{\prime}\right)\right| V_{2} .
$$

But $\rho_{*}\left(P_{1}^{\prime}\right)=0$ and similarly $\rho_{*}\left(K_{E / S} \mid V_{2}\right)=0$ thanks to (a). Furthermore $\delta_{j}-d_{0} t_{j} \geq 0$ by (5.3.3). The assertion of the lemma then follows upon applying $\rho_{*}$.

(5.13) It remains only to carry out the estimates showing that inequalities (3.2.4) and (3.2.5) lead to (2.3.2). Suppose then that $x_{1}$ is a smooth point, and let $\mu=$ mult $_{x}\left(\Delta_{1}\right)$. For $(2.3)$ we need to verify

$$
\left(1-c n-\frac{\sigma}{\sigma_{3}}\right) \sigma_{2} \geq 2-\mu, \quad\left(1-c n-\frac{\sigma}{\sigma_{3}}\right) \sigma_{1} \geq 2-\mu,
$$

so we may assume that $\mu<2$. Since $q>n \sigma_{3} /(1-\sigma)$, we are free to suppose in (3.5) that all the $d_{i}$ satisfy $d_{i}<1-n \sigma_{3} / q(1-\sigma)$. Then

$$
\mu \underset{(5.12)}{\geq} \frac{\left(1-d_{0}\right)}{r}(q-r)>\frac{1}{r}\left(\frac{n \sigma_{3}}{1-\sigma}-r\right)
$$

whence,

$$
\frac{n}{r}<\frac{(\mu+1)(1-\sigma)}{\sigma_{3}}
$$

Therefore,

$$
\left(1-c n-\frac{\sigma}{\sigma_{3}}\right)>1-\frac{n}{r}-\frac{\sigma}{\sigma_{3}} \geq 1-\frac{\mu+1}{\sigma_{3}} .
$$

But the function $(2-x) /\left(1-(x+1) / \sigma_{3}\right)$ is decreasing in $x$ for $x<\sigma_{3}-1$, so we find

$$
\sigma_{2} \underset{(3.2 .4)}{\geq} \frac{2 \sigma_{3}}{\sigma_{3}-1} \geq \frac{2-\mu}{1-(\mu+1) / \sigma_{3}} \underset{(* *)}{\geq} \frac{2-\mu}{\left(1-c n-\sigma / \sigma_{3}\right)} .
$$

The other inequality in $(*)$ is identical. When $x_{1}$ is a rational double point, one argues similarly using instead the second terms on the right sides of (3.2.4) and (3.2.5). We leave details to the reader.

The proof of Theorem 3.2 is now complete.

\section{FujITA'S CONJECTURE ON GLOBAL GENERATION}

We indicate here the modifications necessary to prove Corollary $2^{*}$ in the Introduction. The idea is that the only difficulty occurs when the minimizing component maps to a curve, and $1-c n \approx \frac{1}{4}$. We then argue that by changing slightly the divisor $M$ occuring in (3.9), we can arrange to end up instead in the situation of (4.1).

(6.1) Let $L$ be an ample line bundle on $X$, and let $B=4 L$. We keep notation as in $\S 3$, but we take $X_{0}=X$ and $h=\mathrm{id}$. Thus $x \in X$ is a fixed point, and we need to produce a section of $\mathscr{O}_{X}\left(K_{X}+B\right)$ which is nonvanishing at $x$. 
(6.2) Let $\pi: X_{1} \longrightarrow X$ be the blowing up of $x$, and let $F_{1} \subset X_{1}$ be the exceptional divisor. Since $L$ is ample, we may fix a number $s>0$ such that $\pi^{*}(B)-s F_{1}$ is ample. We next fix $0<\epsilon \ll 1$ such that

$$
\frac{4 \epsilon}{1-\epsilon}<\left(\frac{1-8 \epsilon}{4-8 \epsilon}\right) s
$$

In (3.5), we can then take

$$
\sigma_{3}=4-8 \epsilon, \quad \sigma=\epsilon .
$$

Recall that the resolution $f: Y \longrightarrow X$ constructed in (3.6) factors through the blowing up $\pi$. Denote by $\phi: Y \longrightarrow X_{1}$ the resulting map, and let $F \subset Y$ be the proper transform of $F_{1}$, so that $F$ occurs as one of the exceptional divisors $E_{j}$. Thus the coefficients attached to $F$ in (3.6) are $r(F)=q, b(F)=2$, and $\delta(F)=\delta_{F}$.

(6.3) Observe next that the inequalities (3.2.4) and (3.2.5) needed in $\S 5$ hold with $\sigma_{1}=4-\epsilon_{1}$ and $\sigma_{2}=4-\epsilon_{2}$ for small $\epsilon_{1}$ and $\epsilon_{2}$, but (3.2.3) just fails for $\sigma_{0}=4$. In other words, the only troublesome situation is that occurring in (4.2)-(4.7), when the minimizing component $E$ maps to a curve in $X$. So we may assume we are in that setting. As in $\S 4$, put $\gamma=1-c n-\sigma / \sigma_{3}$. If $\gamma>\frac{1}{4}$, then the argument in (4.5) goes through and we are done. Hence we may suppose that $\gamma \leq \frac{1}{4}$, i.e.,

$$
c n \geq \frac{3-7 \epsilon}{4-8 \epsilon} .
$$

(6.4) We now introduce perturbations of the divisors $R, M$ occurring in (3.9). Specifically, for rational numbers $\lambda, \eta>0$ put

$$
\begin{aligned}
S(\lambda, \eta) & =\sum_{j \geq 0}\left(b_{j}-\lambda r_{j}-\delta_{j}\right) E_{j}-\eta \cdot \phi^{*}\left(F_{1}\right), \\
M(\lambda, \eta) & =f^{*} \mathscr{O}_{X}\left(K_{K}+B\right)-K_{Y}+S(\lambda, \eta) .
\end{aligned}
$$

(Note that the sum is taken over all $E_{j}$, including the minimizing component $\left.E=E_{0}.\right)$ Thus $S(c, 0)=R-E$, and

$$
M(\lambda, \eta) \equiv \phi^{*}\left(\left(1-\lambda n-\frac{\sigma}{\sigma_{3}}\right) \pi^{*} B-\eta F_{1}\right)+A .
$$

(6.5) We claim that in order to prove Corollary $2^{*}$, it suffices to find a rational number $\eta$ such that

$$
\begin{gathered}
0<\eta<\left(1-c n-\frac{\sigma}{\sigma_{3}}\right) s, \\
\operatorname{ord}_{F} S(c, \eta)<-1 .
\end{gathered}
$$

In fact, suppose such $\eta$ exists, and fix $0<\lambda<c$ with $\operatorname{ord}_{F} S(\lambda, \eta)<-1$. Then in the first place, it follows from (6.4.3) and (6.5.1) that $M(\lambda, \eta)$ is ample. Note next that $\operatorname{ord}_{E_{j}} S(\lambda, 0)>-1$ for all $E_{j}$ such that $f\left(E_{j}\right) \ni x$ thanks to 
the definition of $c$ in (3.8). Moreover, by (6.4.1), $\operatorname{ord}_{E_{j}} S(\lambda, \eta)=\operatorname{ord}_{E_{j}} S(\lambda, 0)$ unless $f\left(E_{j}\right)=x$. Therefore, we can write

$$
\left\ulcorner S(\lambda, \eta)^{\urcorner}=P(\lambda, \eta)-N(\lambda, \eta)-T(\lambda, \eta)\right.
$$

where every component of $P(\lambda, \eta)$ is $f$-exceptional, and $N(\lambda, \eta)$ and $T(\lambda, \eta)$ are effective divisors with $\operatorname{supp}(N(\lambda, \eta)) \cap f^{-1}(x)=\varnothing$, and $f(\operatorname{supp}(T(\lambda, \eta)))=$ $x$. Moreover, $T(\lambda, \eta) \neq 0$ since $\operatorname{ord}_{F} S(\lambda, \eta)<-1$. In particular, thinking of $T=T(\lambda, \eta)$ as a subscheme of $Y$, we have $f^{*} \mathscr{O}_{X}\left(K_{X}+B\right) \mid T=\mathscr{O}_{T}$. So as in (3.10) and (3.11) we find that there is a surjective homomorphism

$H^{0}\left(Y, \mathscr{O}_{Y}\left(f^{*}\left(K_{X}+B\right)-N\right)\right) \longrightarrow H^{0}\left(T, \mathscr{O}_{Y}\left(f^{*}\left(K_{X}+B\right)-N\right) \mid T\right)=H^{0}\left(T, \mathscr{O}_{T}\right)$, and we conclude as in (3.12) and (4.1).

(6.6) It remains only to produce the required $\eta$. Recalling from (3.5) that $q>\left(\frac{4-8 \epsilon}{1-\epsilon}\right) n$, we find using $(6.3 .1)$

$$
\begin{aligned}
\operatorname{ord}_{F} S(c, \eta) & =\operatorname{ord}_{F} S(c, 0)-\eta \\
& =2-c q-\delta_{F}-\eta \\
& <2-\frac{3-7 \epsilon}{1-\epsilon}-\eta=-1+\frac{4 \epsilon}{1-\epsilon}-\eta .
\end{aligned}
$$

But $\gamma>1-\frac{3}{4-8 \epsilon}$ by (3.8.1), so we see that it suffices to take $\frac{4 \epsilon}{1-\epsilon}<\eta<\left(\frac{1-8 \epsilon}{4-8 \epsilon}\right) s$, and we are done.

6.7. Remark. In the situation of Theorem $1^{*}$, suppose that $s \geq 0$ is a rational number such that $\pi^{*} B-s \cdot F_{1}$ is big and nef, where $\pi$ is the blowing-up of $X$ at $x \in X$. Then one can show that Theorem $1^{*}$ holds with the last inequality $\sigma_{1} \geq \sigma_{3} /\left(\sigma_{3}-3\right)$ replaced by $\sigma_{1} \geq\left(\sigma_{3}-s\right) /\left(\sigma_{3}-3\right)$. We leave details to the interested reader.

\section{REFERENCES}

[BPV] W. Barth, C. Peters, and A. Van de Ven, Compact complex surfaces, Springer-Verlag, New York, 1984.

[Ben] $\mathrm{X}$. Benveniste, Sur les variétés de dimension 3 de type général dont le diviseur canonique est numériquement positif, Math. Ann. 266 (1984), 479-497.

[Bmb] E. Bombieri, Canonical models of surfaces of general type, Inst. Hautes Études Sci. Publ. Math. 42 (1973), 171-219.

[CKM] H. Clemens, J. Kollár, and S. Mori, Higher dimensional complex geometry, Astérisque 166 (1988).

[Dem] J.-P. Demailly, A numerical criterion for very ample line bundles (to appear).

[EV] H. Esnault and E. Viehweg, Lectures on vanishing theorems, DMV Seminar, bd. 20, Birkhäuser Verlag, Basel, 1993.

[Fuj1] T. Fujita, contribution to Birational geometry of algebraic varieties: Open problems, from the 1988 Katata Syposium of the Taniguchi Foundation.

[Fuj2] _-, On polarized manifolds whose adjoint bundles are not semipositive, Algebraic Geometry, Sendai, 1985 (T. Oda, ed.), Adv. Stud. Pure Math., vol. 10, North-Holland, Amsterdam, 1987, pp. 167-178.

[Fuj3] _ Classification theories of polarized varieties, London Math. Soc. Lecture Note Ser., vol. 155, Cambridge Univ. Press, Cambridge and New York, 1990. 
[Hrt] R. Hartshorne, Generalized divisors on Gorenstein curves and a theorem of Noether, Math. J. Kyoto Univ. 26 (1986), 375-386.

[K] Y. Kawamata, On the finiteness of generators of a pluricanonical ring for a 3-fold of general type, Amer. J. Math. 106 (1984), 1503-1512.

[KMM] Y. Kawamata, K. Matsuda, and K. Matsuki, Introduction to the minimal model problem, Algebraic Geometry, Sendai, 1985 (T. Oda, ed.). Adv. Stud. Pure Math., vol. 10, NorthHolland, Amsterdam, 1987, 283-360.

[Kmf] G. Kempf, Varieties with rational singularities, Proc. of the Lefschetz Centennial Conference, Contemp. Math. 58 (1986), 179-182.

[Kod] K. Kodaira, Pluricanonical systems on algebraic surfaces of general type, J. Math. Soc. Japan 20 (1968), 170-192.

[Kol1] J. Kollár, Vanishing theorems for cohomology groups, Algebraic Geometry, Bowdoin 1985, Proc. Sympos. Pure. Math., vol. 46, Amer. Math. Soc., Providence, RI, 1987, pp. 233-243.

[Kol2] _ Effective base point freeness, preprint.

[Kol3] J. Kollár et al., Flips and abundance for algebraic threefolds (to appear).

[Mat] K. Matsuki, On pluricanonical maps for threefolds of general type, J. Math. Soc. Japan 38 (1986), 339-359.

[Ogu] K. Oguiso, On polarized Calabi-Yau threefolds, J. Fac. Sci. Univ. Tokyo Sect. IA Math. 38 (1991), 395-429.

[Rd] M. Reid, Projective morphisms according to Kawamata, unpublished manuscript, 1983.

[Rdr] I. Reider, Vector bundles of rank 2 and linear systems on algebraic surfaces, Ann. of Math. (2) 127 (1988), 309-316.

[Sak1] F. Sakai, Weil divisors on normal surfaces, Duke Math. J. 51 (1984), 877-887.

[Sak2] _ Reider-Serano's method on normal surfaces, Algebraic Geometry: Proceedings, L'Aquila 1988, Lecture Notes in Math., vol. 1417, Springer-Verlag, New York, 1990, pp. 301-319.

[SS] B. Shiffman and A. Sommese, Vanishing theorems on complex manifolds, Prog. Math., vol. 56, Birkhaüser, Boston, MA, 1985.

[S] V. Shokurov, The non-vanishing theorem, Math. USSR-Izv. 19 (1985), 591-607.

[W1] P. Wilson, Towards birational classification of algebraic varieties, Bull. London Math. Soc. 19 (1987), 1-48.

[W2] - Fano fourfolds of index greater than one, J. Reine Angew. Math. 379, 172-181.

Department of Mathematics, University of Illinois at Chicago, Chicago, Illinois 60680

E-mail address: U22425\%UICVM.BITNET

Department of Mathematics,University of California, Los Angeles, Los Angeles, CalIFORNIA 90024

E-mail address: rkl@math.ucla.edu 